\title{
FINITE QUANTUM GROUPS OVER ABELIAN GROUPS OF PRIME EXPONENT
}

\author{
BY NICOLÁS ANDRUSKIEWITSCH AND HANS-JÜRGEN SCHNEIDER
}

ABSTRACT. - We classify pointed finite-dimensional complex Hopf algebras whose group of group-like elements is abelian of prime exponent $p, p>17$. The Hopf algebras we find are members of a general family of pointed Hopf algebras we construct from Dynkin diagrams. As special cases of our construction we obtain all the Frobenius-Lusztig kernels of semisimple Lie algebras and their parabolic subalgebras. An important step in the classification result is to show that all these Hopf algebras are generated by group-like and skew-primitive elements.

(c) 2002 Éditions scientifiques et médicales Elsevier SAS

RÉSUMÉ. - Nous classifions les algèbres de Hopf complexes de dimension finie dont le groupe des éléments groupoïdaux est abélien d'exposant premier $p, p>17$. Les algèbres de Hopf que nous trouvons appartiennent à une famille d'algèbres de Hopf pointées que nous construisons à partir de diagrammes de Dynkin. Comme cas particuliers de notre construction nous obtenons tous les noyaux de FrobeniusLusztig des algèbres de Lie semi-simples et leurs sous-algèbres paraboliques. Une étape importante dans notre classification consiste à montrer que toutes ces algèbres de Hopf sont engendrées par des éléments groupoïdaux et des éléments primitifs tordus.

(c) 2002 Éditions scientifiques et médicales Elsevier SAS

\section{Introduction}

Since the discovery of quantum groups (Drinfeld, Jimbo) and their finite dimensional variations (Lusztig, Manin), these objects were studied from different points of view and had many applications. The present paper is part of a series where we intend to show that important classes of Hopf algebras are quantum groups and therefore belong to Lie theory.

We will assume that the ground-field $\mathbb{k}$ is algebraically closed of characteristic 0 . One of our main results is the explicit construction of a general family of pointed Hopf algebras from Dynkin diagrams (Theorem 5.17). All the Frobenius-Lusztig kernels and their parabolic subalgebras belong to this family, but in addition we get many new examples. We show that any finite dimensional pointed Hopf algebra with group of prime exponent (greater than 17) is indeed in this family; see our main Theorem below. An important step in the proof follows from another main result (Theorem 7.6), where we show that a large family of finite dimensional pointed Hopf algebras is generated by group-like and skew-primitive elements, giving additional support to a conjecture in [5].

If $A$ is a Hopf algebra, then we denote by $G(A)$ the group of group-like elements of $A$. Recall that $A$ is pointed if $\mathbb{k} G(A)$ is the largest cosemisimple subcoalgebra of $A$, or equivalently if any irreducible $A$-comodule is one-dimensional. 
Let $\Gamma$ be a finite abelian group and $\widehat{\Gamma}$ the group of its characters. We denote the unit element in $\widehat{\Gamma}$ by $\varepsilon$.

To state our main result, we have to introduce some notation. A linking datum $\mathcal{D}$ of finite Cartan type for $\Gamma$ is a collection $\mathcal{D}$ consisting of

a Cartan matrix of finite type $\left(a_{i j}\right)_{1 \leqslant i, j \leqslant \theta}$ of size $\theta \times \theta$ for some $\theta \geqslant 1$, [20], elements $g_{1}, \ldots, g_{\theta} \in \Gamma$, characters $\chi_{1}, \ldots, \chi_{\theta} \in \widehat{\Gamma}$ satisfying

$$
\begin{gathered}
\chi_{i}\left(g_{i}\right) \neq 1, \quad \text { for all } 1 \leqslant i \leqslant \theta, \\
\chi_{j}\left(g_{i}\right) \chi_{i}\left(g_{j}\right)=\chi_{i}\left(g_{i}\right)^{a_{i j}}, \quad \text { for all } 1 \leqslant i, j \leqslant \theta,
\end{gathered}
$$

and a family $\left(\lambda_{i j}\right)_{1 \leqslant i<j \leqslant \theta, i \nsim j}$ of elements in $\mathbb{k}$ such that $\lambda_{i j}$ is arbitrary if $g_{i} g_{j} \neq 1$ and $\chi_{i} \chi_{j}=\varepsilon$, but 0 otherwise.

The elements $\left(\lambda_{i j}\right)_{1 \leqslant i<j \leqslant \theta, i \nsim j}$ are called the linking elements of $\mathcal{D}$.

Here, by $i \sim j$, resp. $i \nsim j, 1 \leqslant i, j \leqslant \theta$ we mean that $i$ and $j$ belong to the same connected component, resp. to different connected components of the Dynkin diagram corresponding to $\left(a_{i j}\right)$.

Now we fix a prime $p>2$ and a natural number $s$. We consider finite abelian groups of the form $\Gamma(s):=(\mathbb{Z} /(p))^{s}$.

Let $\mathcal{D}$ be a linking datum of finite Cartan type for $\Gamma(s)$ with Cartan matrix $\left(a_{i j}\right)_{1 \leqslant i, j \leqslant \theta}$ and linking elements $\left(\lambda_{i j}\right)_{1 \leqslant i<j \leqslant \theta, i \nsim j}$.

We define the algebra $\mathfrak{u}(\mathcal{D})$ by generators $a_{1}, \ldots, a_{\theta}, y_{1}, \ldots, y_{s}$ and relations

$$
\begin{aligned}
y_{h}^{p} & =1, \quad y_{m} y_{h}=y_{h} y_{m}, \quad \text { for all } 1 \leqslant m, h \leqslant s, \\
g a_{j} & =\chi_{j}(g) a_{j} y_{h}, \quad \text { for all } g \in \Gamma, 1 \leqslant j \leqslant \theta, \\
\left(\operatorname{ad} a_{i}\right)^{1-a_{i j}} a_{j} & =0, \quad \text { for all } 1 \leqslant i \neq j \leqslant \theta, i \sim j, \\
a_{i} a_{j}-\chi_{j}\left(g_{i}\right) a_{j} a_{i} & =\lambda_{i j}\left(1-g_{i} g_{j}\right), \quad \text { for all } 1 \leqslant i<j \leqslant \theta, i \nsim j ; \\
a_{\alpha}^{p} & =0, \quad \text { for all } \alpha \in \Phi^{+} .
\end{aligned}
$$

To formulate these relations we used the following natural interpretation of elements $g \in \Gamma$ as words in the generators $y_{h}, 1 \leqslant h \leqslant s$. Let $Y_{h}, 1 \leqslant h \leqslant s$, be a $\mathbb{Z} /(p)$-basis of $\Gamma$, and write $g=Y_{1}^{t_{1}} \cdots Y_{s}^{t_{s}}$, where $t_{1}, \ldots, t_{s}$ are natural numbers. Then in the relations above replace $g$ by the formal expression $y_{1}^{t_{1}} \cdots y_{s}^{t_{s}}$.

In (1.5), ad $a_{i}$ is the adjoint action of $a_{i}$, that is for all $x \in \mathfrak{u}(\mathcal{D})$,

$$
\left(\operatorname{ad} a_{i}\right) x=a_{i(1)} x \mathcal{S}\left(a_{i(2)}\right)=a_{i} x-g_{i} x g_{i}^{-1} a_{i} .
$$

In this way the left hand side of (1.5) is meant as a well-defined expression in the generators.

In (1.7), $\Phi^{+}$is the set of positive roots of the root system associated to the Cartan matrix $\left(a_{i j}\right)$; the "root vectors" $a_{\alpha}$ are defined in Section 4.1 below.

Our main theorem is

THEOREM 1.8. - (a) Let $\mathcal{D}$ be a linking datum of finite Cartan type for $\Gamma(s)$ with Cartan matrix $\left(a_{i j}\right)$, and assume that $p>3$ if $\left(a_{i j}\right)$ has a connected component of type $G_{2}$. Then $\mathfrak{u}(\mathcal{D})$ has a unique Hopf algebra structure determined by

$$
\Delta y_{h}=y_{h} \otimes y_{h}, \quad \Delta a_{i}=a_{i} \otimes 1+g_{i} \otimes a_{i}, \quad \text { for all } 1 \leqslant h \leqslant s, 1 \leqslant i \leqslant \theta .
$$

The Hopf algebra $\mathfrak{u}(\mathcal{D})$ is pointed, $G(\mathfrak{u}(\mathcal{D})) \simeq \Gamma(s)$ and $\operatorname{dim} \mathfrak{u}(\mathcal{D})=p^{s+\mid \Phi^{+}}$.

(b) Let $p>17$. Let $\mathcal{A}$ be a pointed finite-dimensional Hopf algebra such that $G(\mathcal{A}) \simeq \Gamma(s)$. Then there exists a linking datum $\mathcal{D}$ of finite Cartan type for $\Gamma(s)$ such that $\mathcal{A} \simeq \mathfrak{u}(\mathcal{D})$. 
Remarks 1.10. - (i) In Section 5 we define the notion of a "linking datum" for a general finite abelian group $\Gamma$. In the situation of the main theorem it is always possible to reduce to the case of linking data with all entries $\lambda_{i j}$ equal to 0 or 1 . Thus it follows from Theorem 1.8 that there are only finitely many isomorphism classes of finite dimensional Hopf algebras with fixed coradical $\mathbb{k} \Gamma(s)$. For more general finite abelian groups, this is no longer true $[4,8,16]$.

(ii) The dimensions of the Hopf algebras in Theorem 1.8 are very special numbers. This phenomenon is shown in general for arbitrary finite groups $\Gamma$ in Theorem 7.9.

(iii) Let $\left(a_{i j}\right)_{1 \leqslant i, j \leqslant \theta}$ be a finite Cartan matrix. The problem of actually finding all the collections $g_{i} \in \Gamma(s), \chi_{i} \in \widehat{\Gamma(s)}, 1 \leqslant i \leqslant \theta$, such that (1.1) and (1.2) hold has been discussed in [5]. It can be stated as the problem of finding all the solutions of a system of algebraic equations over $\mathbb{Z} /(p)$ and it is in principle solvable. Note that in particular

$$
\theta \leqslant 2 s \frac{p-1}{p-2}
$$

see [5, Prop. 8.3].

(iv) The question of finding all the possible linking elements attached to a fixed collection $g_{i}, \chi_{i}, 1 \leqslant i \leqslant \theta,\left(a_{i j}\right)_{1 \leqslant i, j \leqslant \theta}$, is also of combinatorial nature, see Section 5, and also [13]. Once these two problems are solved effectively, the isomorphism classes of the Hopf algebras $\mathfrak{u}(\mathcal{D})$ can be determined using [5, Prop. 6.3], [6, Lemma 1.2].

(v) As a consequence of Theorem 1.8 one obtains the complete classification of all finite dimensional pointed Hopf algebras with group of group-likes $\Gamma(1)=\mathbb{Z} /(p), p \neq 5,7$. It is the list given in [5, Theorem 1.3] plus the Frobenius-Lusztig kernels as described in [4]. Indeed, replacing in the proof of Theorem 1.8 [5, Cor. 1.2] by [5, Th. 1.3] we get the classification for all primes $p \neq 5$ or 7 , in view of Theorem 6.8 and [6, Lemma 4.2]. The only cases not covered are $p=5$, type $B_{2}$ and $p=7$, type $G_{2}$. This result was independently obtained by Musson [28] using different methods starting from our previous article [5].

(vi) Up to now, the determination of all finite dimensional pointed Hopf algebras $A$ with $G(A) \simeq \Gamma$, for a fixed group $\Gamma$, was known only for $\Gamma=\mathbb{Z} /(2)$ [29]. Other classification results of pointed Hopf algebras are known for some fixed dimension $d: d=p^{2}$ is easy and follows from $[29,30] ; d=p^{3}$ was done in [4], and by different methods in [10,36]; $d=p^{4}$ in [6] (and does not seem to be possible via the other methods); $d=16$ in [11], $d=32$ in [17]; results on the case when $\Gamma$ has exponent 2 can be found in [2].

(vii) The classification of all coradically graded pointed Hopf algebras of dimension $p^{5}$ was obtained in [18]. It is not difficult to deduce the classification of all pointed Hopf algebras of dimension $p^{5}$ using Theorem 1.8 and results in [6].

(viii) The Hopf algebras $\mathfrak{u}(\mathcal{D})$ can be defined for any Cartan datum of finite type $\mathcal{D}$ of an arbitrary finite abelian group. Part (a) of Theorem 1.8 is a special case of the general Theorem 5.17. For suitable choices of $\mathcal{D}$, the Frobenius-Lusztig kernels and their parabolic subalgebras are of the form $\mathfrak{u}(\mathcal{D})$. See Example 5.12. Otherwise Theorem 5.17 provides many new examples of finite dimensional Hopf algebras arising from exotic linking data.

(ix) The results of this paper heavily depend on our paper [5] and on previous work on quantum groups $[21,22,33,34,12,27]$.

Conventions. Our reference for the theory of Hopf algebras is [26]. The notation for Hopf algebras is standard: $\Delta, \mathcal{S}, \epsilon$, denote respectively the comultiplication, the antipode, the counit; we use Sweedler's notation but dropping the summation symbol.

If $C$ is a coalgebra then $G(C)$ denotes the set of its group-like elements and $C_{0} \subset C_{1} \subset \ldots$ its coradical filtration. So that $C_{0}$ is the coradical of $C$. As usual, $\mathcal{P}_{g, h}(C)$ denotes the space 
of $(g, h)$-skew primitives of $C, g, h \in G(C)$. If $C$ has a distinguished group-like 1, then we set $\mathcal{P}(C)=\mathcal{P}_{1,1}(C)$, the space of primitive elements of $C$.

If $A$ is an algebra and $\left(x_{i}\right)_{i \in I}$ is a family of elements of $A$ then $\mathbb{k}\left\langle x_{i}\right\rangle_{i \in I}$ or simply $\mathbb{k}\left\langle x_{i}\right\rangle$, resp. $\left\langle x_{i}\right\rangle_{i \in I}$ or $\left\langle x_{i}\right\rangle$ denotes the subalgebra, resp. the two-sided ideal, generated by the $x_{i}$ 's.

Let $H$ be a Hopf algebra. A Yetter-Drinfeld module over $H$ is a vector space $V$ provided with structures of left $H$-module and left $H$-comodule such that $\delta(h . v)=h_{(1)} v_{(-1)} \mathcal{S} h_{(3)} \otimes h_{(2)} \cdot v_{(0)}$. We denote by ${ }_{H}^{H} \mathcal{Y D}$ the (braided) category of Yetter-Drinfeld modules over $H$.

Assume that $H=\mathbb{k} \Gamma$ where $\Gamma$ is a finite abelian group. We denote ${ }_{\Gamma}^{\Gamma} \mathcal{Y} \mathcal{D}:={ }_{H}^{H} \mathcal{Y} \mathcal{D}$. Let $g \in \Gamma$, $\chi \in \widehat{\Gamma}$ and $V$ a module, resp. a comodule, resp. a Yetter-Drinfeld module, over $\Gamma$. Then we denote $V^{\chi}=\{v \in V: h . v=\chi(h) v, \forall h \in \Gamma\}$, resp. $V_{g}=\{v \in V: \delta(v)=g \otimes v\}$, resp. $V_{g}^{\chi}:=V_{g} \cap V^{\chi}$. If $V$ is a locally finite Yetter-Drinfeld module, then $V=\bigoplus_{g \in \Gamma, \chi \in \widehat{\Gamma}} V_{g}^{\chi}$. Conversely, a vector space $V$ provided with a direct sum decomposition $V=\bigoplus_{g \in \Gamma, \chi \in \widehat{\Gamma}} V_{g}^{\chi}$ has an evident YetterDrinfeld module structure.

\section{Outline of the paper and proof of the main result}

Theorem 1.8 follows from Theorems 4.5, 5.17, 6.8, 6.10 and Corollary 7.7 in the present article, along the guidelines proposed in [4]. We give now the proof of Theorem 1.8 assuming those results which hold over arbitrary finite abelian groups. This section serves also as a guide to the different sections of the paper.

\subsection{The proof}

Let $\mathcal{A}$ be a finite dimensional pointed Hopf algebra with $G(\mathcal{A}) \simeq \Gamma(s)$. Let

$$
\operatorname{gr} \mathcal{A}:=\bigoplus_{n \geqslant 0} \operatorname{gr} \mathcal{A}(n)
$$

where gr $\mathcal{A}(0)=\mathcal{A}_{0}, \operatorname{gr} \mathcal{A}(n)=\mathcal{A}_{n} / \mathcal{A}_{n-1}$, if $n>0$ be the graded coalgebra associated to the coradical filtration of $\mathcal{A}$. Then $\operatorname{gr} \mathcal{A}$ is a graded Hopf algebra [26] and both the inclusion $\iota: \mathcal{A}_{0} \hookrightarrow \operatorname{gr} \mathcal{A}$ and the graded projection $\pi: \operatorname{gr} \mathcal{A} \rightarrow \mathcal{A}_{0}$ are Hopf algebra maps. Let

$$
R:=\operatorname{gr} \mathcal{A}^{c o \pi}=\{x \in \operatorname{gr} \mathcal{A}:(\operatorname{id} \otimes \pi) \Delta(x)=x \otimes 1\}
$$

it is a graded braided Hopf algebra in ${ }_{\Gamma(s)}^{\Gamma(s)} \mathcal{Y D}$ with the grading inherited from gr $\mathcal{A}$ :

$$
R=\bigoplus_{n \geqslant 0} R(n), \quad R(n):=R \cap \operatorname{gr} \mathcal{A}(n) .
$$

Notice that $\operatorname{gr} \mathcal{A}$ can be reconstructed from $R$ as a biproduct:

$$
\operatorname{gr} \mathcal{A} \simeq R \# \mathbb{k} \Gamma(s) .
$$

The braided Hopf algebra $R$ is called the diagram of $\mathcal{A}$. One has

$$
\begin{aligned}
& R(0)=\mathbb{k} 1, \\
& R(1)=\mathcal{P}(R),
\end{aligned}
$$

$4^{\mathrm{e}}$ SÉRIE - TOME $35-2002-\mathrm{N}^{\circ} 1$ 
and we know from Corollary 7.7 below that

$R$ is generated as an algebra by $\mathrm{R}(1)$.

Let $V:=R(1)$; it is a Yetter-Drinfeld submodule of $R$. Since $R$ satisfies (2.1), (2.2) and (2.3) we know that $R \simeq \mathfrak{B}(V)$ is a Nichols algebra, see Section 3.2. Now there exists a basis $x_{1}, \ldots, x_{\theta}$ of $V$ and $g_{1}, \ldots, g_{\theta} \in \Gamma(s), \chi_{1}, \ldots, \chi_{\theta} \in \widehat{\Gamma(s)}$ such that $x_{i} \in V_{g_{i}}^{\chi_{i}}, 1 \leqslant i \leqslant \theta$. Since $\mathcal{A}$ is finite dimensional, $\chi_{i}\left(g_{i}\right) \neq 1$ for all $i\left[4\right.$, Lemma 3.1] and there is a finite Cartan matrix $\left(a_{i j}\right)_{1 \leqslant i, j \leqslant \theta}$ such that (1.2) holds [5, Cor. 1.2].

To give an explicit description of $\mathfrak{B}(V)$, we introduce root vectors in $\mathfrak{B}(V)$ generalizing the root vectors defined in [21]. We note that Lusztig's root vectors can be described up to a nonzero scalar as an iterated braided commutator of simple root vectors. We then define the root vectors in the general case by exactly the same iterated braided commutator with respect to our more general braiding. As one of our main results, we obtain a presentation by generators and relations and a PBW basis for $\mathfrak{B}(V)$ from the corresponding Theorem for Frobenius-Lusztig kernels, using Drinfeld's twisting essentially in the same way as in [5]. See Theorem 4.5. We can then deduce part (a) of Theorem 1.8. For connected Dynkin diagrams it is a consequence of Theorem 4.5; in the non-connected case we apply the idea of twisting the algebra structure by a 2 -cocycle which is given by a Hopf algebra map [14]. See Theorem 5.17.

It follows from Theorem 4.5 that gr $\mathcal{A}$ can be presented as an algebra by generators $y_{1}, \ldots, y_{s}$ (homogeneous of degree 0 ) and $x_{1}, \ldots, x_{\theta}$ (homogeneous of degree 1 ), and relations

$$
\begin{aligned}
y_{h}^{p} & =1, \quad y_{m} y_{h}=y_{h} y_{m}, \quad \text { for all } 1 \leqslant m, h \leqslant s, \\
y_{h} x_{j} & =\chi_{j}\left(y_{h}\right) x_{j} y_{h}, \quad \text { for all } 1 \leqslant h \leqslant s, 1 \leqslant j \leqslant \theta, \\
\left(\operatorname{ad} x_{i}\right)^{1-a_{i j}} x_{j} & =0, \quad \text { for all } 1 \leqslant i \neq j \leqslant \theta \\
x_{\alpha}^{p} & =0, \quad \text { for all } \alpha \in \Phi^{+} ;
\end{aligned}
$$

and where the Hopf algebra structure is determined by

$$
\Delta y_{h}=y_{h} \otimes y_{h}, \quad \Delta x_{i}=x_{i} \otimes 1+g_{i} \otimes x_{i}, \quad \text { for all } 1 \leqslant h \leqslant s, 1 \leqslant i \leqslant \theta .
$$

By [4, Lemma 5.4], we can choose $a_{i} \in \mathcal{P}_{g_{i}, 1}(\mathcal{A})^{\chi_{i}}$ such that the class of $a_{i}$ in $\operatorname{gr} \mathcal{A}(1)$ coincides with $x_{i}$. We also keep the notation $y_{j}$ for the generators of $G(\mathcal{A})$. It is clear that relations (1.3) and (1.4) hold. Now relations (1.5) and (1.6), resp. (1.7), hold because of Theorem 6.8, resp. Lemma 6.9.

The Theorem now follows from Theorem 6.10.

\subsection{The general case}

There are several obstructions to extend Theorem 1.8 to general finite abelian groups. First, it is open whether the diagram of a finite dimensional pointed Hopf algebra is generated in degree one, i.e. when it is a Nichols algebra; second, there are finite dimensional Nichols algebras which are not of Cartan type [29].

For liftings of gr $A$ when $R$ is a Nichols algebra of Cartan type, the quantum Serre relations of connected vertices in general still hold as we show in Theorem 6.8 below; however the powers of the root vectors are not necessarily 0 . We should have $a_{\alpha}^{N_{\alpha}}=u_{\alpha} \in \mathbb{k} G(A)$; the determination of $u_{\alpha}$ when $\alpha$ is a non-simple root was done in [6] for type $A_{2}$, in [9] for type $B_{2}$, and in [7] for type $A_{n}$ for any $n$ (up to some exceptional cases concerning the orders of the roots of unity). 


\section{Braided Hopf algebras}

\subsection{Biproducts}

Let $R$ be a braided Hopf algebra in ${ }_{H}^{H} \mathcal{Y D}$; this means that $R$ is an algebra and a coalgebra in ${ }_{H}^{H} \mathcal{Y D}$ and that the comultiplication $\Delta_{R}: R \rightarrow R \otimes R$ is an algebra map when in $R \otimes R$ the multiplication twisted by the braiding $c$ is considered; in addition $R$ admits an antipode. To avoid confusions we use the following variant of Sweedler's notation for the comultiplication of $R: \Delta_{R}(r)=r^{(1)} \otimes r^{(2)}$. Let $A=R \# H$ be the biproduct or bosonization of $R$ [24], [31]. Recall that the multiplication and comultiplication of $A$ are given by

$$
(r \# h)(s \# f)=r\left(h_{(1)} . s\right) \# h_{(2)} f, \quad \Delta(r \# h)=r^{(1)} \#\left(r^{(2)}\right)_{(-1)} h_{(1)} \otimes\left(r^{(2)}\right)_{(0)} \# h_{(2)} .
$$

The maps $\pi: A \rightarrow H$ and $\iota: H \rightarrow A, \pi(r \# h)=\epsilon(r) h, \iota(h)=1 \# h$, are Hopf algebra homomorphisms; we have $R=\{a \in A$ : $(\operatorname{id} \otimes \pi) \Delta(a)=a \otimes 1\}$. Conversely, let $A, H$ be Hopf algebras provided with Hopf algebra homomorphisms $\pi: A \rightarrow H$ and $\iota: H \rightarrow A$. Then $R=\{a \in A:($ id $\otimes \pi) \Delta(a)=a \otimes 1\}$ is a braided Hopf algebra in ${ }_{H}^{H} \mathcal{Y D}$. The action . of $H$ on $R$ is the restriction of the adjoint action (composed with $\iota$ ) and the coaction is ( $\pi \otimes \mathrm{id}) \Delta ; R$ is a subalgebra of $A$ and the comultiplication is $\Delta_{R}(r)=r_{(1)} \iota \pi \mathcal{S}\left(r_{(2)}\right) \otimes r_{(3)}$. These constructions are inverse to each other. We shall mostly omit $\iota$ in what follows.

Let $\vartheta: A \rightarrow R$ be the map given by $\vartheta(a)=a_{(1)} \pi \mathcal{S}\left(a_{(2)}\right)$. Then

$$
\vartheta(a b)=a_{(1)} \vartheta(b) \pi \mathcal{S}\left(a_{(2)}\right),
$$

for all $a, b \in A$ and $\vartheta(h)=\varepsilon(h)$ for all $h \in H$; therefore, for all $a \in A, h \in H$, we have $\vartheta(a h)=\vartheta(a) \varepsilon(h)$ and

$$
\vartheta(h a)=h . \vartheta(a)=\vartheta\left(h_{(1)} a \pi \mathcal{S}\left(h_{(2)}\right)\right) .
$$

Notice also that $\vartheta$ induces a coalgebra isomorphism $A / A H^{+} \simeq R$. In fact, the isomorphism $A \rightarrow R \# H$ can be expressed explicitly as

$$
a \mapsto \vartheta\left(a_{(1)}\right) \# \pi\left(a_{(2)}\right), \quad a \in A .
$$

If $A$ is a Hopf algebra, the well-known adjoint representation ad of $A$ on itself is given by $\operatorname{ad} x(y)=x_{(1)} y \mathcal{S}\left(x_{(2)}\right)$. If $R$ is a braided Hopf algebra in ${ }_{H}^{H} \mathcal{Y D}$ then there is also a braided adjoint representation $\operatorname{ad}_{c}$ of $R$ on itself given by

$$
\operatorname{ad}_{c} x(y)=\mu(\mu \otimes \mathcal{S})(\mathrm{id} \otimes c)(\Delta \otimes \mathrm{id})(x \otimes y),
$$

where $\mu$ is the multiplication and $c \in \operatorname{End}(R \otimes R)$ is the braiding. Note that if $x \in \mathcal{P}(R)$ then the braided adjoint representation of $x$ is just

$$
\operatorname{ad}_{c} x(y)=\mu(\mathrm{id}-c)(x \otimes y)=:[x, y]_{c} .
$$

The element $[x, y]_{c}$ defined by the second equality for any $x$ and $y$, regardless of whether $x$ is primitive, will be called a braided commutator.

When $A=R \# H$, then for all $b, d \in R$,

$$
\operatorname{ad}_{(b \# 1)}(d \# 1)=\left(\operatorname{ad}_{c} b(d)\right) \# 1 .
$$




\subsection{Nichols algebras}

Let $H$ be a Hopf algebra and let $R=\bigoplus_{n \in \mathbb{N}} R(n)$ be a graded braided Hopf algebra in ${ }_{H}^{H} \mathcal{Y D}$. We say that $R$ is a Nichols algebra if 2.1,2.2 and 2.3 hold, cf. [29,5,3]. A Nichols algebra $R$ is uniquely determined by the Yetter-Drinfeld module $\mathcal{P}(R)$; given a Yetter-Drinfeld module $V$, there exists a unique (up to isomorphism) Nichols algebra $R$ with $\mathcal{P}(R) \simeq V$. It will be denoted $\mathfrak{B}(V)$. In fact, the kernel of the canonical map $\varpi: T(V) \rightarrow \mathfrak{B}(V)$ can be described in several different ways. For instance, $\operatorname{Ker} \varpi=\bigoplus_{n \geqslant 0} \operatorname{Ker} \mathbf{S}_{n}$ where $\mathbf{S}_{n}$ is the "quantum symmetrizer" defined from the braiding $c$; so that $\mathfrak{B}(V)$ is a "quantum shuffle algebra" and as algebra and coalgebra only depends on the braiding $c: V \otimes V \rightarrow V \otimes V$. See [29,37,23,33-35].

Let $H=\mathbb{k} \Gamma$ where $\Gamma$ is a finite abelian group. Let $V$ be a finite dimensional YetterDrinfeld module over $\Gamma$. Then there exist a basis $x_{1}, \ldots, x_{\theta}$ of $V$ and elements $g_{1}, \ldots, g_{\theta} \in \Gamma$, $\chi_{1}, \ldots, \chi_{\theta} \in \widehat{\Gamma}$ such that

$$
x_{j} \in V_{g_{j}}^{\chi_{j}}, \quad \text { for all } 1 \leqslant j \leqslant \theta .
$$

In what follows we shall only consider Yetter-Drinfeld modules $V$ such that $\chi_{i}\left(g_{i}\right) \neq 1$, $1 \leqslant i \leqslant \theta$. The braiding $c$ is given with respect to the basis $x_{i} \otimes x_{j}$ by $c\left(x_{i} \otimes x_{j}\right)=b_{i j} x_{j} \otimes x_{i}$, where

$$
\left(b_{i j}\right)_{1 \leqslant i, j \leqslant \theta}=\left(\chi_{j}\left(g_{i}\right)\right)_{1 \leqslant i, j \leqslant \theta} .
$$

Remark 3.6. - Let $V$, resp. $\widetilde{V}$, be a finite dimensional Yetter-Drinfeld module over $\Gamma$, resp. $\widetilde{\Gamma}$, with a basis $x_{1}, \ldots, x_{\theta}$ such that $x_{i} \in V_{g_{i}}^{\chi_{i}}$, resp. with a basis $\tilde{x}_{1}, \ldots, \tilde{x}_{\theta}$ such that $\tilde{x}_{i} \in V_{\tilde{g}_{i}} \tilde{\chi}_{i}$. Assume that $\chi_{i}\left(g_{j}\right)=\tilde{\chi}_{i}\left(\tilde{g}_{j}\right)$ for all $1 \leqslant i, j \leqslant \theta$. Then there exists a unique algebra and coalgebra isomorphism $\mathfrak{B}(V) \rightarrow \mathfrak{B}(\tilde{V})$ such that $x_{i} \mapsto \tilde{x}_{i}$ for all $1 \leqslant i \leqslant \theta$.

DEFINITION 3.7. - We shall say that a braiding given by a matrix $\mathbf{b}=\left(b_{i j}\right)_{1 \leqslant i, j \leqslant \theta}$ whose entries are roots of unity is of Cartan type if for all $i, j, b_{i i} \neq 1$ and there exists $a_{i j} \in \mathbb{Z}$ such that

$$
b_{i j} b_{j i}=b_{i i}^{a_{i j}} .
$$

The integers $a_{i j}$ are uniquely determined by the following rules:

- If $i=j$ we take $a_{i i}=2$;

- if $i \neq j$, we select the unique $a_{i j}$ such that $-\operatorname{ord} b_{i i}<a_{i j} \leqslant 0$.

Then $\left(a_{i j}\right)$ is a generalized Cartan matrix [20]. We shall say a Yetter-Drinfeld module $V$ is of Cartan type, resp. finite Cartan type, if its corresponding braiding is of Cartan type, resp. the same plus the matrix $\left(a_{i j}\right)$ is of finite type.

\subsection{The twisting functor}

Let $H$ be a Hopf algebra and let $F$ be an invertible element in $H \otimes H$ such that

$$
F_{12}(\Delta \otimes \mathrm{id}) F=F_{23}(\mathrm{id} \otimes \Delta) F, \quad(\varepsilon \otimes \mathrm{id})(F)=1=(\mathrm{id} \otimes \varepsilon)(F) .
$$

Then $H_{F}$, the same algebra $H$ but with the comultiplication $\Delta_{F}:=F \Delta F^{-1}$, is again a Hopf algebra [15]. We shall write $F=F^{1} \otimes F^{2}, F^{-1}=G^{1} \otimes G^{2}$; the new comultiplication will be denoted by $\Delta_{F}(h)=h_{(1, F)} \otimes h_{(2, F)}$.

Let now $R$ be a braided Hopf algebra in ${ }_{H}^{H} \mathcal{Y D}$, let $A=R \# H$ be its bosonization and consider the Hopf algebra $A_{F}$. It follows from the definitions that $\pi: A_{F} \rightarrow H_{F}$ and $\iota: H_{F} \rightarrow A_{F}$ are 
also Hopf algebra homomorphisms. Hence

$$
R_{F}:=\left\{a \in A_{F}:(\mathrm{id} \otimes \pi) \Delta_{F}(a)=a \otimes 1\right\}
$$

is a braided Hopf algebra in the category ${ }_{H_{F}}^{H_{F}} \mathcal{Y} \mathcal{D}$. We consider the corresponding map $\vartheta_{F}$ and define $\psi: R \rightarrow R_{F}$ by

$$
\psi(r)=\vartheta_{F}(r), \quad r \in R .
$$

The map $\psi$ was defined in [5] in the case $H=\mathbb{k} \Gamma$ is the group algebra of a finite abelian group. The following lemma generalizes [5, Lemma 2.3]; part (iii), new even for $H=\mathbb{k} \Gamma$, will be needed in the sequel.

LEMMA 3.10. - (i) $\psi$ is an isomorphism of $H$-modules. (Recall that $H=H_{F}$ as algebras.)

(ii) If $r, s \in R$ then

$$
\psi(r s)=F^{1} \cdot \psi(r) F^{2} \cdot \psi(s) .
$$

(iii) If $r \in R$ then

$$
\Delta_{R_{F}} \psi(r)=F^{1} \cdot \psi\left(r^{(1)}\right) \otimes F^{2} \cdot \psi\left(r^{(2)}\right) .
$$

(iv) If $R$ is a graded braided Hopf algebra, then $R_{F}$ also is and $\psi$ is a graded map. If $R$ is a coradically graded braided Hopf algebra (resp. a Nichols algebra), then $R_{F}$ also is.

Proof. - (i) follows from (3.2): $\psi(h . r)=\vartheta_{F}(h . r)=\vartheta_{F}(h r)=h . \vartheta_{F}(r)=h . \psi(r)$. Now we prove (ii):

$$
\begin{aligned}
\psi(r s) & =\vartheta_{F}(r s)=r_{(1, F)} \vartheta_{F}(s) \pi\left(\mathcal{S}_{F}\left(r_{(2, F)}\right)\right) \\
& =r_{(1, F)} \pi\left(\mathcal{S}_{F}\left(r_{(2, F)}\right)\right) \pi\left(r_{(3, F)}\right) \vartheta_{F}(s) \pi\left(\mathcal{S}_{F}\left(r_{(4, F)}\right)\right) \\
& =\psi\left(r_{(1, F)}\right) \pi\left(r_{(2, F)}\right) \cdot \psi(s)=\psi\left(F^{1} r_{(1)} G^{1}\right) \pi\left(F^{2} r_{(2)} G^{2}\right) \cdot \psi(s) \\
& =\psi\left(F^{1} r_{(1)}\right) \varepsilon\left(G^{1}\right) \pi\left(F^{2} r_{(2)} G^{2}\right) \cdot \psi(s)=F^{1} \cdot \psi\left(r_{(1)}\right) \pi\left(F^{2}\right) \pi\left(r_{(2)}\right) \cdot \psi(s) \\
& =F^{1} \cdot \psi(r) \pi\left(F^{2}\right) \cdot \psi(s),
\end{aligned}
$$

as claimed. Here we have used (3.1), the definitions and (3.8). For the proof of (iii), we first observe that, if $r \in R$, then

$$
\psi\left(r^{(1)}\right) \otimes \psi\left(r^{(2)}\right)=\vartheta_{F}\left(r_{(1)} \pi \mathcal{S}\left(r_{(2)}\right)\right) \otimes \vartheta_{F}\left(r_{(3)}\right)=\psi\left(r_{(1)}\right) \otimes \psi\left(r_{(2)}\right) .
$$

Using that $\vartheta_{F}$ is a coalgebra map, (3.2) and (3.13), we conclude that

$$
\begin{aligned}
\Delta_{R_{F}} \psi(r) & =\Delta_{R_{F}} \vartheta_{F}(r)=\vartheta_{F}\left(r_{(1, F)}\right) \otimes \vartheta_{F}\left(r_{(2, F)}\right) \\
& =\vartheta_{F}\left(F^{1} r_{(1)} G^{1}\right) \otimes \vartheta_{F}\left(F^{2} r_{(2)} G^{2}\right)=\vartheta_{F}\left(F^{1} r_{(1)}\right) \otimes \vartheta_{F}\left(F^{2} r_{(2)}\right) \\
& =F^{1} . \vartheta_{F}\left(r_{(1)}\right) \otimes F^{2} . \vartheta_{F}\left(r_{(2)}\right)=F^{1} . \psi\left(r^{(1)}\right) \otimes F^{2} . \psi\left(r^{(2)}\right) .
\end{aligned}
$$

The proof of (iv) has no difference with the proof of the analogous statement in [5, Lemma 2.3].

We now consider the special case when $H=\mathbb{k} \Gamma, \Gamma$ a finite abelian group. Let $\omega: \widehat{\Gamma} \times \widehat{\Gamma} \rightarrow \mathbb{k}^{\times}$ be a 2 -cocycle, i.e. $\omega(\tau, 1)=\omega(1, \tau)=1$ and $\omega(\tau, \zeta) \omega(\tau \zeta, \eta)=\omega(\tau, \zeta \eta) \omega(\zeta, \eta)$. The cocycle $\omega$ allows to define a map $\Psi: \widehat{\Gamma} \times \Gamma \rightarrow \Gamma$ by

$$
\langle\tau, \Psi(\chi, g)\rangle=\omega(\tau, \chi) \omega(\chi, \tau)^{-1}\langle\tau, g\rangle, \quad \tau \in \widehat{\Gamma} .
$$


We identify $H$ with the Hopf algebra $\mathbb{k ^ { \Gamma }}$ of functions on the group $\widehat{\Gamma}$; we denote by $\delta_{\tau} \in H$ the function given by $\delta_{\tau}(\zeta)=\delta_{\tau, \zeta}, \tau, \zeta \in \widehat{\Gamma}$. Then $\delta_{\tau}=\frac{1}{|\Gamma|} \sum_{g \in \Gamma}\left\langle\tau, g^{-1}\right\rangle g$. Let $F \in H \otimes H$ be given by

$$
F=\sum_{\tau, \zeta \in \widehat{\Gamma}} \omega(\tau, \zeta) \delta_{\tau} \otimes \delta_{\zeta}
$$

Then $F$ satisfies (3.8); note that $H=H_{F}$. Let now $R$ be a braided Hopf algebra in $\Gamma_{\Gamma}^{\Gamma} \mathcal{Y} \mathcal{D}$; we can consider the Hopf algebras $A=R \# \mathbb{k} \Gamma$ and $A_{F}$, the braided Hopf algebra $R_{F} \in{ }_{\Gamma}^{\Gamma} \mathcal{Y} \mathcal{D}$ and the map $\psi: R \rightarrow R_{F}$. We have

$$
\psi(r)=\sum_{\tau \in \widehat{\Gamma}} \omega(\chi, \tau)^{-1} r \# \delta_{\tau}, \quad r \in R^{\chi} ; \quad \psi\left(R_{g}^{\chi}\right)=R_{\Psi(\chi, g)}^{\psi} .
$$

See [5, Lemma 2.3]. Note that (3.11) is now $\psi(r s)=\omega(\chi, \tau) \psi(r) \psi(s), r \in R^{\chi}, s \in R^{\tau}$.

LEMMA 3.15. - If $r \in P(R)_{g}^{\chi}$ and $s \in R^{\tau}$ then

$$
\psi\left([r, s]_{c}\right)=\omega(\chi, \tau)[\psi(r), \psi(s)]_{c} .
$$

Proof. - We have

$$
\begin{aligned}
\psi\left([r, s]_{c}\right) & =\psi(r s-\tau(g) s r) \\
& =\omega(\chi, \tau) \psi(r) \psi(s)-\omega(\tau, \chi) \tau(g) \psi(s) \psi(r) \\
& =\omega(\chi, \tau)(\psi(r) \psi(s)-\langle\tau, \Psi(\chi, g)\rangle \psi(s) \psi(r)) \\
& =\omega(\chi, \tau)[\psi(r)), \psi(s)]_{c},
\end{aligned}
$$

where we used (3.14).

Remark 3.17. - It is possible to show that $(\psi \otimes \psi) c(r \otimes s)=F . c_{F}(\psi(r) \otimes \psi(s))$, for all $r \in R_{g}^{\chi}, s \in R^{\tau}$.

From the previous considerations and Lemma 3.10 we immediately get

Proposition 3.18. - Let $R$ be an algebra in $\Gamma_{\Gamma}^{\Gamma} \mathcal{Y} \mathcal{D},\left(x_{i}\right)_{i \in I}$ a family of elements of $R$, $x_{i} \in R_{g_{i}}^{\chi_{i}}$ for some $g_{i} \in \Gamma, \chi_{i} \in \widehat{\Gamma}$. Then:

(i) $\psi\left(\mathbb{k}\left\langle x_{i}\right\rangle\right)=\mathbb{k}\left\langle\psi\left(x_{i}\right)\right\rangle, \psi\left(\left\langle x_{i}\right\rangle\right)=\left\langle\psi\left(x_{i}\right)\right\rangle$.

(ii) If $R$ has a presentation by generators $x_{i}$ and relations $t_{j}$, where also the $t_{j}$ 's are homogeneous then $R_{F}$ has a presentation by generators $\psi\left(x_{i}\right)$ and relations $\psi\left(t_{j}\right)$.

(iii) If $x_{i}$ is central and $\omega\left(\chi_{i}, \tau\right)=\omega\left(\tau, \chi_{i}\right)$ for all $\tau$ such that $R^{\tau} \neq 0$, then $\psi\left(x_{i}\right)$ is central.

\section{Root vectors and Quantum Serre relations}

\subsection{Root vectors}

In this section, we assume the following situation:

We fix a finite abelian group $\Gamma$, a finite Cartan matrix $\left(a_{i j}\right)_{1 \leqslant i, j \leqslant \theta}$ and $g_{1}, \ldots, g_{\theta} \in \Gamma$, $\chi_{1}, \ldots, \chi_{\theta} \in \widehat{\Gamma}$ such that (1.1) and (1.2) hold. Let $d_{1}, \ldots, d_{\theta} \in\{1,2,3\}$ such that $d_{i} a_{i j}=d_{j} a_{j i}$ for all $i, j$. We set $q_{i}=\chi_{i}\left(g_{i}\right), N_{i}$ the order of $q_{i}$. We assume, for all $i$ and $j$, that the order of $\chi_{i}\left(g_{j}\right)$ is odd, and that $N_{i}$ is not divisible by 3 if $i$ belongs to a connected component of type $G_{2}$. 
Let $\mathcal{X}$ be the set of connected components of the Dynkin diagram corresponding to $\left(a_{i j}\right)$. We assume that for each $I \in \mathcal{X}$, there exist $c_{I}, d_{I}$ such that $I=\left\{j: c_{I} \leqslant j \leqslant d_{I}\right\}$; that is, after reordering the Cartan matrix is a matrix of blocks corresponding to the connected components. Let $I \in \mathcal{X}$ and $i \sim j$ in $I$; then $N_{i}=N_{j}$, hence $N_{I}:=N_{i}$ is well defined. Let $\Phi_{I}$, resp. $\Phi_{I}^{+}$, be the root system, resp. the subset of positive roots, corresponding to the Cartan matrix $\left(a_{i j}\right)_{i, j \in I}$; then $\Phi=\bigcup_{I \in \mathcal{X}} \Phi_{I}$, resp. $\Phi^{+}=\bigcup_{I \in \mathcal{X}} \Phi_{I}^{+}$is the root system, resp. the subset of positive roots, corresponding to the Cartan matrix $\left(a_{i j}\right)_{1 \leqslant i, j \leqslant \theta}$. Let $\alpha_{1}, \ldots, \alpha_{\theta}$ be the set of simple roots.

Let $\mathcal{W}_{I}$ be the Weyl group corresponding to the Cartan matrix $\left(a_{i j}\right)_{i, j \in I}$; we identify it with a subgroup of the Weyl group $\mathcal{W}$ corresponding to the Cartan matrix $\left(a_{i j}\right)$. We fix a reduced decomposition of the longest element $\omega_{0, I}$ of $\mathcal{W}_{I}$ in terms of simple reflections. Then we obtain a reduced decomposition of the longest element $\omega_{0}=s_{i_{1}} \ldots s_{i_{P}}$ of $\mathcal{W}$ from the expression of $\omega_{0}$ as product of the $\omega_{0, I}$ 's in some fixed order of the components, say the order arising from the order of the vertices. Therefore $\beta_{j}:=s_{i_{1}} \ldots s_{i_{j-1}}\left(\alpha_{i_{j}}\right)$ is a numeration of $\Phi^{+}$.

We fix a finite dimensional Yetter-Drinfeld module $V$ over $\Gamma$ with a basis $x_{1}, \ldots, x_{\theta}$ with $x_{i} \in V_{g_{i}}^{\chi_{i}}, 1 \leqslant i \leqslant \theta$.

Major examples of modules of Cartan type are the Frobenius-Lusztig kernels. Let $N>1$ be an odd natural number and let $q \in \mathbb{k}$ be a primitive $N$ th root of 1 , not divisible by 3 in case $\left(a_{i j}\right)$ has a component of type $G_{2}$. Let $\mathbb{G}=\mathbb{Z} /(N)^{\theta}=\left\langle e_{1}\right\rangle \oplus \cdots \oplus\left\langle e_{\theta}\right\rangle$; let $\eta_{j} \in \widehat{\mathbb{G}}$ be the unique character such that $\langle\eta(j), e(i)\rangle=q^{d_{i} a_{i j}}$. Let $\mathbb{V}$ be a Yetter-Drinfeld module over $\mathbb{G}$ with a basis $X_{1}, \ldots, X_{\theta}$ such that

$$
X_{i} \in \mathbb{V}_{\eta_{i}}^{e_{i}}, \quad \text { for all } 1 \leqslant i \leqslant \theta .
$$

We denote by $\mathfrak{c}$ the braiding of $\mathbb{V}$. Lusztig defined root vectors $X_{\alpha} \in \mathfrak{B}(\mathbb{V}), \alpha \in \Phi^{+}$[22]. One can see from [23] that, up to a non-zero scalar, each root vector can be written as an iterated braided commutator in some sequence $X_{\ell_{1}}, \ldots, X_{\ell_{a}}$ of simple root vectors such as $\left[\left[X_{\ell_{1}},\left[X_{\ell_{2}}, X_{\ell_{3}}\right]_{\mathfrak{c}}\right]_{\mathfrak{c}},\left[X_{\ell_{4}}, X_{\ell_{5}}\right]_{\mathfrak{c}}\right]_{\mathfrak{c}}$. This can also be seen in the situation in [32].

We now fix for each $\alpha \in \Phi^{+}$such a representation of $X_{\alpha}$ as an iterated braided commutator. In the general case of our $V$, we define root vectors $x_{\alpha}$ in the tensor algebra $T(V), \alpha \in \Phi^{+}$, as the same formal iteration of braided commutators in the elements $x_{1}, \ldots, x_{\theta}$ instead of $X_{1}, \ldots, X_{\theta}$ but with respect to the braiding $c$ given by the general matrix $\left(\chi_{j}\left(g_{i}\right)\right)$. Note that each $x_{\alpha}$ is homogeneous and has the same degree as $X_{\alpha}$, where we mean the degree in the sense of [23]. Also,

$$
x_{\alpha} \in T(V)_{g_{\alpha}}^{\chi_{\alpha}},
$$

where $g_{\alpha}=g_{1}^{b_{1}} \cdots g_{\theta}^{b_{\theta}}, \chi_{\alpha}=\chi_{1}^{b_{1}} \cdots \chi_{\theta}^{b_{\theta}}$, where $\alpha=b_{1} \alpha_{1}+\cdots+b_{\theta} \alpha_{\theta}$.

THEOREM 4.2. - The Nichols algebra $\mathfrak{B}(\mathbb{V})$ is presented by generators $X_{i}, 1 \leqslant i \leqslant \theta$, and relations

$$
\begin{aligned}
\operatorname{ad}_{c}\left(X_{i}\right)^{1-a_{i j}}\left(X_{j}\right) & =0, \quad \text { for all } i \neq j, \\
X_{\alpha}^{N} & =0, \quad \text { for all } \alpha \in \Phi^{+} .
\end{aligned}
$$

Moreover, the following elements constitute a basis of $\mathfrak{B}(\mathbb{V})$ :

$$
X_{\beta_{1}}^{h_{1}} X_{\beta_{2}}^{h_{2}} \cdots X_{\beta_{P}}^{h_{P}}, \quad \text { for all } 0 \leqslant h_{j} \leqslant N-1,1 \leqslant j \leqslant P .
$$

Proof. - It follows from results of Lusztig [21,22], Rosso [33,34] and Müller [27] that $\mathfrak{B}(\mathbb{V})$ is the positive part of the so-called Frobenius-Lusztig kernel corresponding to the Cartan matrix $\left(a_{i j}\right)$. See [5, Th. 3.1] for details. The presentation by generators and relations follows from the 
considerations in the last paragraph of p. 15 and the first paragraph of p. 16 in [1] referring to $[12, \S 19$, Corollary in p. 120]. The statement about the basis is [21,22].

\subsection{Nichols algebras of Cartan type}

We can now prove the first main result of the present paper, describing $\mathfrak{B}(V)$ by generators and relations when $V$ is of finite Cartan type, improving [5, Th. 1.1(i)]. As in loc. cit., we use repeatedly Remark 3.6.

THEOREM 4.5. - The Nichols algebra $\mathfrak{B}(V)$ is presented by generators $x_{i}, 1 \leqslant i \leqslant \theta$, and relations

$$
\begin{aligned}
\operatorname{ad}_{c}\left(x_{i}\right)^{1-a_{i j}}\left(x_{j}\right) & =0, \quad \text { for all } i \neq j, \\
x_{\alpha}^{N_{I}} & =0, \quad \text { for all } \alpha \in \Phi_{I}^{+}, I \in \mathcal{X} .
\end{aligned}
$$

Moreover, the following elements constitute a basis of $\mathfrak{B}(V)$ :

$$
x_{\beta_{1}}^{h_{1}} x_{\beta_{2}}^{h_{2}} \cdots x_{\beta_{P}}^{h_{P}}, \quad \text { for all } 0 \leqslant h_{j} \leqslant N_{I}-1 \text {, if } \beta_{j} \in I, 1 \leqslant j \leqslant P .
$$

Proof. - (a) Let us first assume that the braiding is symmetric, that is $\chi_{i}\left(g_{j}\right)=\chi_{j}\left(g_{i}\right)$ for all $i, j$. By [5, Lemma 4.2] we can assume moreover that the Cartan matrix $\left(a_{i j}\right)$ is connected. From our assumptions on the orders of the $\chi_{i}\left(g_{j}\right)$ we then conclude that the braiding has the form $\chi_{j}\left(g_{i}\right)=q^{d_{i} a_{i j}}$ for all $i, j$ where $q$ is a root of unity of order $N=\chi_{i}\left(g_{i}\right)$. See [5, Lemma 4.3]. Hence the Theorem follows directly from Theorem 4.2 and Remark 3.6.

(b) In the case of an arbitrary braiding we know from Lemma 4.1 of [5] that there exists a finite abelian group $\mathbb{G}$ satisfying:

- The braiding $c$ of $V$ can be realized from a Yetter-Drinfeld module structure over $\mathbb{G}$ that we continue denoting by $V$, cf. Remark 3.6.

- There exists a cocycle $\omega: \widehat{\mathbb{G}} \times \widehat{\mathbb{G}} \rightarrow \mathbb{k}^{\times}$with corresponding $F \in \mathbb{k} \mathbb{G} \otimes \mathbb{k} \mathbb{G}$ such that the braiding of $V_{F}$ is symmetric. Let $\psi: \mathfrak{B}(V) \rightarrow \mathfrak{B}\left(V_{F}\right)$ be the isomorphism having the same meaning as in (3.9).

- The braiding of $V_{F}$ is given in the basis $\psi\left(x_{i}\right) \otimes \psi\left(x_{j}\right)$ by a matrix $\left(b_{i j}^{F}\right)$ such that $b_{i i}^{F}=\chi_{i}\left(g_{i}\right)$ and the order of $\left(b_{i j}^{F}\right)$ is again odd for all $i$ and $j$.

If $\varpi: T(V) \rightarrow \mathfrak{B}(V), \varpi_{F}: T\left(V_{F}\right) \rightarrow \mathfrak{B}\left(V_{F}\right)$ denote the canonical maps, then we have a commutative diagram

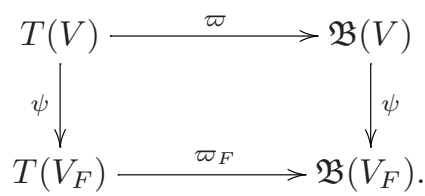

Clearly, $\psi(\operatorname{Ker} \varpi)=\operatorname{Ker} \varpi_{F}$; if $\left(r_{j}\right)_{j \in J}$ is a set of generators of the ideal Ker $\varpi$ with $r_{j} \in T(V)_{h_{j}}^{\eta_{j}}$ then by Proposition $3.18\left(\psi\left(r_{j}\right)\right)_{j \in J}$ is a set of generators of the ideal Ker $\varpi_{F}$. By the symmetric case (a), we know the generators of $\operatorname{Ker} \varphi_{F}$. Let us denote $X_{i}:=\psi\left(x_{i}\right)$. Then by Lemma 3.15 and (3.11), we have $\psi\left(\operatorname{ad}_{c}\left(x_{i}\right)^{1-a_{i j}}\left(x_{j}\right)\right)=u_{i j} \operatorname{ad}_{c}\left(X_{i}\right)^{1-a_{i j}}\left(X_{j}\right)$ and $\psi\left(x_{\alpha}^{N_{I}}\right)=u_{\alpha} X_{\alpha}^{N_{I}}, \alpha \in \Phi_{I}^{+}$where $u_{i j}, u_{\alpha}$ are non-zero scalars. This implies the first claim of the theorem. The second follows in a similar way.

Let $\widehat{\mathfrak{B}}(V)$ be the braided Hopf algebra in ${ }_{\Gamma}^{\Gamma} \mathcal{Y} \mathcal{D}$ generated by $x_{1}, \ldots, x_{\theta}$ with relations (4.6), where the $x_{i}$ 's are primitive. Let $\mathcal{K}(V)$ be the subalgebra of $\widehat{\mathfrak{B}}(V)$ generated by $x_{\alpha}^{N_{I}}, \alpha \in \Phi_{I}^{+}$, $I \in \mathcal{X}$; it is a Yetter-Drinfeld submodule of $\widehat{\mathfrak{B}}(V)$. 
THEOREM 4.8. - $\mathcal{K}(V)$ is a braided Hopf subalgebra in ${ }_{\Gamma}^{\Gamma} \mathcal{Y D}$ of $\widehat{\mathfrak{B}}(V)$.

Proof. - (a) As in the proof of Theorem 4.5 we first assume that the braiding is symmetric. If $i \neq j$, then $\chi_{j}\left(g_{i}\right) \chi_{i}\left(g_{j}\right)=1$ and hence the corresponding Serre relation (4.6) says that $x_{i} x_{j}=x_{j} x_{i}$. Thus, we can easily reduce to the connected case. In such case, $\chi_{j}\left(g_{i}\right)=q^{d_{i} a_{i j}}$ as before and the Theorem is shown in [12].

(b) In the general case, we change the group as in the proof of Theorem 4.5. The isomorphism $\psi: T(V) \rightarrow T\left(V_{F}\right)$ respects the Serre relations up to non-zero scalars by Lemma 3.15. Also, it maps subcoalgebras stable under the action of the group to subcoalgebras by Lemma 3.10(iii). We conclude from (a) that $\mathcal{K}(V)$ is a subcoalgebra of $\widehat{\mathfrak{B}}(V)$.

\section{Linking datum and glueing of connected components}

\subsection{Linking datum}

In this section, we fix a finite abelian group $\Gamma$, a finite Cartan matrix $\left(a_{i j}\right)_{1 \leqslant i, j \leqslant \theta}$ and $g_{1}, \ldots, g_{\theta} \in \Gamma, \chi_{1}, \ldots, \chi_{\theta} \in \widehat{\Gamma}$ such that (1.1) and (1.2) hold. We preserve the conventions and hypotheses from Section 4.

DEFinition 5.1. - We say that two vertices $i$ and $j$ are linkable (or that $i$ is linkable to $j$ ) if

$$
\begin{aligned}
& i \nsim j, \\
& g_{i} g_{j} \neq 1 \text { and } \\
& \chi_{i} \chi_{j}=1 .
\end{aligned}
$$

If $i$ is linkable to $j$, then $\chi_{i}\left(g_{j}\right) \chi_{j}\left(g_{i}\right)=1$ by (5.2); it follows then from (5.4) that

$$
\chi_{j}\left(g_{j}\right)=\chi_{i}\left(g_{i}\right)^{-1} .
$$

LemmA 5.6. - Assume that $i$ and $k$, resp. $j$ and $\ell$, are linkable. Then $a_{i j}=a_{k \ell}, a_{j i}=a_{\ell k}$. In particular, a vertex $i$ can not be linkable to two different vertices $j$ and $h$.

Proof. - If $a_{i \ell} \neq 0$ then $a_{i j}=a_{j i}=0$ (otherwise $j \sim \ell$ ) and $a_{k \ell}=a_{\ell k}=0$ (otherwise $i \sim k$ ). If $a_{j k} \neq 0$ then $a_{i j}=a_{j i}=0$ (otherwise $i \sim k$ ) and $a_{k \ell}=a_{\ell k}=0$ (otherwise $j \sim \ell$ ). Assume that $a_{i \ell}=0=a_{j k}$. Then

$$
\begin{aligned}
\chi_{i}\left(g_{i}\right)^{a_{i j}} & =\chi_{i}\left(g_{j}\right) \chi_{j}\left(g_{i}\right)=\chi_{k}^{-1}\left(g_{j}\right) \chi_{\ell}^{-1}\left(g_{i}\right)=\chi_{j}\left(g_{k}\right) \chi_{i}\left(g_{\ell}\right) \\
& =\chi_{\ell}^{-1}\left(g_{k}\right) \chi_{k}^{-1}\left(g_{\ell}\right)=\chi_{k}\left(g_{k}\right)^{-a_{k \ell}}=\chi_{i}\left(g_{i}\right)^{a_{k \ell}} .
\end{aligned}
$$

Then $N_{i}$ divides $a_{i j}-a_{k \ell}$ and analogously, $N_{k}$ divides $a_{i j}-a_{k \ell}$. So that $a_{i j}=a_{k \ell}$ by the assumptions on the order of $N_{i}$ and $N_{k}$; by symmetry, $a_{j i}=a_{\ell k}$. Assume that a vertex $i$ is linkable to $j$ and $h$. Then $2=a_{i i}=a_{j h}$, so $j=h$.

A linking datum of finite Cartan type for $\Gamma$ is a collection

$$
\mathcal{D}=\mathcal{D}\left(\Gamma,\left(a_{i j}\right)_{1 \leqslant i, j \leqslant \theta},\left(g_{i}\right)_{1 \leqslant i \leqslant \theta},\left(\chi_{j}\right)_{1 \leqslant j \leqslant \theta},\left(\lambda_{i j}\right)_{1 \leqslant i<j \leqslant \theta, i \nsim j}\right)
$$

where $\left(a_{i j}\right)$ is a Cartan matrix of finite type, $\left(g_{i}\right),\left(\chi_{j}\right)$ are elements as above, and $\left(\lambda_{i j}\right)_{1 \leqslant i<j \leqslant \theta, i \nsim j}$ are elements in $\mathbb{k}$ such that $\lambda_{i j}$ is arbitrary if $i$ and $j$ are linkable but 0 otherwise. The elements $\lambda_{i j}$ are called the linking elements of $\mathcal{D}$. Given a linking datum, we say that two vertices $i$ and $j$ are linked if $\lambda_{i j} \neq 0$. 
This definition generalizes part of the definition of compatible datum in [4, Section 5]. We shall represent a linking datum by the Dynkin diagram of the Cartan matrix $\left(a_{i j}\right)$ joining linked vertices by a dotted line. To have a complete picture we add the pair $\left(g_{i}, \chi_{i}\right)$ below the vertex $i$.

DEFINITION 5.7. - Let us fix a decomposition $\Gamma=\left\langle Y_{1}\right\rangle \oplus \cdots \oplus\left\langle Y_{s}\right\rangle$; let $M_{h}$ denote the order of $Y_{h}, 1 \leqslant h \leqslant s$. Let $\mathcal{D}=\mathcal{D}\left(\Gamma,\left(a_{i j}\right)_{1 \leqslant i, j \leqslant \theta},\left(g_{i}\right)_{1 \leqslant i \leqslant \theta},\left(\chi_{j}\right)_{1 \leqslant j \leqslant \theta},\left(\lambda_{i j}\right)_{1 \leqslant i<j \leqslant \theta, i \nsim j}\right)$ be a linking datum. We denote by $\mathfrak{u}(\mathcal{D})$ the algebra presented by generators $a_{1}, \ldots, a_{\theta}, y_{1}, \ldots, y_{s}$ and relations

$$
y_{h}^{M_{h}}=1, \quad y_{m} y_{h}=y_{h} y_{m}, \quad \text { for all } 1 \leqslant m, h \leqslant s,
$$

(1.4), (1.5), (1.6) and

$$
a_{\alpha}^{N_{I}}=0, \quad \text { for all } \alpha \in \Phi_{I}^{+}, I \in \mathcal{X} .
$$

Remark 5.10. - In the preceding definition, one could consider only linking data with $\lambda_{i j}=1$ or 0 . Indeed, one can replace the generator $a_{i}$ by $\lambda_{i j}^{-1} a_{i}$ whenever $\lambda_{i j} \neq 0$ for some $j$ which is unique by Lemma 5.6. The other relations do not change since they are homogeneous in the $a_{i}$ 's. However, in the more general case where the relations (5.9) have a non-zero right side, one needs general linking data.

Example 5.11. - Here is a linking datum where all the connected components are points:

Example 5.12. - Let $\mathbf{B}:=\left(b_{i j}\right)_{1 \leqslant i, j \leqslant R}$ be a finite Cartan matrix, $0 \leqslant M \leqslant R$ and $q \in \mathbb{k}$ a root of unity of order $N$; we assume $N$ is odd, and prime to 3 if $\mathbf{B}$ contains a component of type $G_{2}$. Let $d_{1}, \ldots, d_{R}$ be integers in $\{1,2,3\}$ such that $d_{i} b_{i j}=d_{j} b_{j i}$. Let $\theta=R+M$, $\widetilde{\mathbf{B}}:=\left(b_{i j}\right)_{1 \leqslant i, j \leqslant M}$ and $\mathbf{A}=\left(a_{i j}\right)$ be the Cartan matrix

$$
\mathbf{A}=\left(\begin{array}{cc}
\mathbf{B} & 0 \\
0 & \widetilde{\mathbf{B}}
\end{array}\right)
$$

Let $\Gamma=(\mathbb{Z} /(N))^{R}, g_{1}, \ldots, g_{R}$ the canonical basis of $\Gamma$ and $\chi_{1}, \ldots, \chi_{R}$ be the character given by $\chi_{i}\left(g_{j}\right)=q^{d_{i} b_{i j}}$; let $g_{R+j}=g_{j}, \chi_{R+j}=\chi_{j}^{-1}, 1 \leqslant j \leqslant M$. Note that $j$ and $j+R$ are linkable, $1 \leqslant j \leqslant M$. Finally, let $\lambda_{j, j+R}=1$ if $1 \leqslant j \leqslant M$ and 0 otherwise; then $\left(\lambda_{i j}\right)_{1 \leqslant i<j \leqslant \theta}$ is a linking datum for $\Gamma,\left(a_{i j}\right), g_{1}, \ldots, g_{\theta}$ and $\chi_{1}, \ldots, \chi_{\theta}$. The Hopf algebra $\mathfrak{u}(\mathcal{D})$ with comultiplication determined by (1.9) is the parabolic part of a Frobenius-Lusztig kernel. Since the numeration of the Dynkin diagram is so far arbitrary, any such parabolic appears in this way.

Example 5.13. - Here are some exotic examples of linking data:

Take 4 copies of $A_{3}$ and label the vertices such that $\{1,2,3\},\{4,5,6\},\{7,8,9\}$ and $\{10,11,12\}$ are the connected components. Then link 3 with 4,6 with 7,9 with 10 and 12 with 1 . It is possible to realize this linking over $\mathbb{Z} /(N)^{12}$ for any odd $N$; the corresponding braiding will be symmetric in each component, that is, the corresponding subalgebra is the "Borel part" of a Frobenius-Lusztig kernel. More examples arise considering more copies of more general components.

See [13] for a combinatorial description of all linkings of Dynkin diagrams. 


\subsection{Altering the multiplication by a cocycle}

The following variation of Drinfeld's twisting was stated by Doi: if $H$ is a Hopf algebra and $\sigma: H \times H \rightarrow \mathbb{k}$ is an invertible 2-cocycle, so that

$$
\begin{aligned}
\sigma\left(x_{(1)}, y_{(1)}\right) \sigma\left(x_{(2)} y_{(2)}, z\right) & =\sigma\left(y_{(1)}, z_{(1)}\right) \sigma\left(x, y_{(2)} z_{(2)}\right), \\
\sigma(1,1) & =1,
\end{aligned}
$$

for all $x, y, z \in H$, then $H_{\sigma}$ - the same $H$ but with the multiplication ${ }_{\sigma}$ below - is again a Hopf algebra, where

$$
x \cdot \sigma y=\sigma\left(x_{(1)}, y_{(1)}\right) x_{(2)} y_{(2)} \sigma^{-1}\left(x_{(3)}, y_{(3)}\right) .
$$

LEMMA 5.14. - [14] Let $U, B$ be Hopf algebras.

(a) Let $\tau: U \otimes B \rightarrow \mathbb{k}$ be a bilinear map such that for all $u, v \in U, a, b \in B$

(1) $\tau(u v, a)=\tau\left(u, a_{(1)}\right) \tau\left(v, a_{(2)}\right)$,

(2) $\tau(u, a b)=\tau\left(u_{(1)}, b\right) \tau\left(u_{(2)}, a\right)$,

(3) $\tau(1, a)=\varepsilon(a)$,

(4) $\tau(u, 1)=\varepsilon(u)$.

Let $H$ be the tensor product Hopf algebra $H=U \otimes B$ and let $\sigma: H \otimes H \rightarrow \mathbb{k}$ be the bilinear map $\sigma(u \otimes a, v \otimes b)=\varepsilon(u) \tau(v, a) \varepsilon(b)$, for all $u, v \in U, a, b \in B$. Then $\tau$ is convolution invertible with inverse given by $\tau^{-1}(v, a)=\varphi(\mathcal{S} v)(a)=\varphi(v)\left(\mathcal{S}^{-1} a\right) ; \sigma$ is an invertible 2-cocycle - with inverse $\sigma^{-1}(u \otimes a, v \otimes b)=\varepsilon(u) \tau^{-1}(v, a) \varepsilon(b)$, for all $u, v \in U, a, b \in B$-and consequently $H_{\sigma}$ is a Hopf algebra.

(b) Assume that $B$ is finite dimensional and let $\varphi: U \rightarrow\left(B^{*}\right)^{\text {cop }}$ be a Hopf algebra homomorphism. Then $\tau: U \otimes B \rightarrow \mathbb{k}, \tau(v, a)=\varphi(v)(a)$, is invertible - with inverse given by $\tau^{-1}(v, a)=\varphi(\mathcal{S} v)(a)=\varphi(v)\left(\mathcal{S}^{-1} a\right)$, and satisfies $1,2,3$ and 4 . Reciprocally, given such $\tau$ there is a unique such $\varphi$.

The following result is probably known. We include it for completeness.

Lemma 5.15. - Let $U, B$ and $\tau$ be as in the preceding lemma. Assume that

- $U$ is generated as an algebra by skew-primitive elements $u_{i}, i \in I$, and group-like elements $g_{k}, k \in K$, which in addition generate $G(U)$ as a monoid;

- $B$ is generated as an algebra by skew-primitive elements $b_{j}, j \in J$, and group-like elements $h_{\ell}, \ell \in L$, which in addition generate $G(B)$ as a monoid.

Let $A$ be an algebra and let $\alpha: U \rightarrow A, \beta: B \rightarrow A$ be algebra maps and let $\gamma:(U \otimes B)_{\sigma} \rightarrow A$ be given by $\gamma(u \otimes b)=\alpha(u) \beta(b)$ for all $u \in U, b \in B$. Then $\gamma$ is an algebra map if and only if

$$
\tau\left(u_{(1)}, b_{(1)}\right) \alpha\left(u_{(2)}\right) \beta\left(b_{(2)}\right)=\beta\left(b_{(1)}\right) \alpha\left(u_{(1)}\right) \tau\left(u_{(2)}, b_{(2)}\right),
$$

whenever $u$, resp. $b$, belongs to the family $u_{i}, i \in I$ or $g_{k}, k \in K$, resp. $b_{j}, j \in J$ or $h_{\ell}, \ell \in L$.

Proof. - (Sketch). Clearly, $\gamma$ is an algebra map if and only if (5.16) holds for all $u \in U, b \in B$. It follows also easily that (5.16) holds when $u=1$, or $b=1$, or $u \in G(U)$ and $b \in G(B)$. Next, let $u, v \in U$ and $b, c \in B$ be arbitrary elements; one can then check that (5.16) holds for $u v$ and $b c$ if it holds for all the possibilities $u_{(1)}$ and $c_{(1)} ; u_{(2)}$ and $b_{(1)} ; v_{(1)}$ and $c_{(2)} ; v_{(2)}$ and $b_{(2)}$. From this observation and the hypothesis the lemma follows. 


\subsection{Glueing of connected components}

In this subsection, we fix a linking datum

$$
\mathcal{D}=\mathcal{D}\left(\Gamma,\left(a_{i j}\right)_{1 \leqslant i, j \leqslant \theta},\left(g_{i}\right)_{1 \leqslant i \leqslant \theta},\left(\chi_{j}\right)_{1 \leqslant j \leqslant \theta},\left(\lambda_{i j}\right)_{1 \leqslant i<j \leqslant \theta, i \nsim j}\right)
$$

of finite Cartan type. Let $\mathcal{A}=\mathfrak{u}(\mathcal{D})$ be the algebra defined in Definition 5.7.

THEOREM 5.17. - (a) There exists a unique Hopf algebra structure on $\mathcal{A}$ determined by (1.9).

(b) The dimension of $\mathcal{A}$ is $|\Gamma| \prod_{I \in \mathcal{X}} N_{I}^{\left|\Phi_{I}^{+}\right|}$.

Proof. - By induction on the number of connected components. Here is the first step:

LEMmA 5.18. - Theorem (5.17) is true if the Dynkin diagram corresponding to $\left(a_{i j}\right)_{1 \leqslant i, j \leqslant \theta}$ is connected.

Proof. - Let $V=\bigoplus_{1 \leqslant i \leqslant \theta} V_{g_{i}}^{\chi_{i}}$ be a Yetter-Drinfeld module over $\Gamma$ with $\operatorname{dim} V_{g_{i}}^{\chi_{i}}=1$ and pick $x_{i} \in V_{g_{i}}^{\chi_{i}}-0$. By Theorem 4.5 and the formulas for the biproduct, there exists a unique algebra map $\mathcal{F}: \mathcal{A} \rightarrow \mathfrak{B}(V) \# \mathbb{k} \Gamma$ such that $\mathcal{F}\left(a_{i}\right)=x_{i} \# 1, \mathcal{F}\left(y_{t}\right)=1 \# y_{t}$. Also, by Theorem 4.5 again, there are algebra maps $\mathcal{G}_{1}: \mathfrak{B}(V) \rightarrow \mathcal{A}, \mathcal{G}_{2}: \mathbb{k} \Gamma \rightarrow \mathcal{A}$ such that $\mathcal{G}_{1}\left(x_{i}\right)=a_{i}, \mathcal{G}_{2}\left(y_{t}\right)=y_{t}$. Let $\mathcal{G}: \mathfrak{B}(V) \# \mathbb{k} \Gamma \rightarrow \mathcal{A}, \mathcal{G}(x \# u)=\mathcal{G}_{1}(x) \mathcal{G}_{2}(u), x \in \mathfrak{B}(V), u \in \mathbb{k} \Gamma$; then $\mathcal{G}$ is an algebra map by (1.4). It is clear now that $\mathcal{F}$ is an isomorphism with inverse $\mathcal{G}$; thus $\mathcal{A}$ is a Hopf algebra and has the desired dimension by the dimension formula in Theorem 4.5.

For the rest of this proof we assume: there exists $\tilde{\theta}<\theta$ such that $i \sim j$, resp. $i \nsim h$, if $1 \leqslant i \leqslant \tilde{\theta}$ and $1 \leqslant j \leqslant \tilde{\theta}$, resp. $\tilde{\theta}<h \leqslant \theta$. Let $J=\{1, \ldots, \tilde{\theta}\} \in \mathcal{X}$. Let $\Upsilon:=\left\langle Z_{1}\right\rangle \oplus \cdots \oplus\left\langle Z_{\tilde{\theta}}\right\rangle$, where the order of $Z_{i}$ is the least common multiple of ord $g_{i}$ and ord $\chi_{i}, 1 \leqslant i \leqslant \tilde{\theta}$. Let $\eta_{j}$ be the unique character of $\Upsilon$ such that $\eta_{j}\left(Z_{i}\right)=\chi_{j}\left(g_{i}\right), 1 \leqslant i \leqslant \tilde{\theta}, 1 \leqslant j \leqslant \tilde{\theta}$. This is well defined because ord $g_{i}$ divides ord $Z_{i}$ for all $i$.

- $\mathcal{B}:=\mathfrak{u}\left(\mathcal{D}_{1}\right)$, with $\mathcal{D}_{1}=\mathcal{D}\left(\Gamma,\left(a_{i j}\right)_{\tilde{\theta}<i, j \leqslant \theta},\left(g_{i}\right)_{\tilde{\theta}<i \leqslant \theta},\left(\chi_{j}\right)_{\tilde{\theta}<j \leqslant \theta},\left(\lambda_{i j}\right)_{\tilde{\theta}<i<j \leqslant \theta, i \nsim j}\right)$, with generators $b_{\tilde{\theta}+1}, \ldots, b_{\theta}$ (instead of the $a_{i}$ 's) and $y_{1}, \ldots, y_{s}$;

- $\mathcal{U}:=\mathfrak{u}\left(\mathcal{D}_{2}\right)$, with $\mathcal{D}_{2}=\mathcal{D}\left(\Upsilon,\left(a_{i j}\right)_{1 \leqslant i, j \leqslant \tilde{\theta}},\left(Z_{i}\right)_{1 \leqslant i \leqslant \tilde{\theta}},\left(\eta_{j}\right)_{1 \leqslant j \leqslant \tilde{\theta}},\left(\lambda_{i j}\right)_{1 \leqslant i<j \leqslant \tilde{\theta}, i \nsim j}\right)$, with generators $u_{1}, \ldots, u_{\tilde{\theta}}$ (instead of the $a_{i}$ 's) and $z_{1}, \ldots, z_{\tilde{\theta}}$.

Note that the linking datum of $\mathcal{U}$ is empty since $J$ is connected. By the induction hypothesis, $\operatorname{dim} \mathcal{B}=|\Gamma| \prod_{I \in \mathcal{X}, I \neq J} N_{I}^{\left|\Phi_{I}^{+}\right|}$and $\operatorname{dim} \mathcal{U}=|\Upsilon| N_{J}^{\left|\Phi_{J}^{+}\right|}$.

LEMMA 5.19. - (a) For each $i, 1 \leqslant i \leqslant \tilde{\theta}$, there exists a unique character $\gamma_{i}: \mathcal{B} \rightarrow \mathbb{k}$ such that

$$
\gamma_{i}\left(y_{k}\right)=\chi_{i}\left(y_{k}\right), \quad \gamma_{i}\left(b_{j}\right)=0
$$

$1 \leqslant k \leqslant s, \tilde{\theta}+1 \leqslant j \leqslant \theta$

(b) Let $\left(\tilde{\lambda}_{i j}\right)_{1 \leqslant i<j \leqslant \theta, i \nsim j}$ be an arbitrary linking datum. For each $i, 1 \leqslant i \leqslant \tilde{\theta}$, there exists a unique $\left(\varepsilon, \gamma_{i}\right)$-derivation $\delta_{i}: \mathcal{B} \rightarrow \mathbb{k}$ such that

$$
\delta_{i}\left(y_{k}\right)=0, \quad \delta_{i}\left(b_{j}\right)=\tilde{\lambda}_{i j}
$$

$1 \leqslant k \leqslant s, \tilde{\theta}+1 \leqslant j \leqslant \theta$.

(c) There exists a unique Hopf algebra map $\varphi: \mathcal{U} \rightarrow\left(\mathcal{B}^{*}\right)^{\text {cop }}$ such that

$$
\varphi\left(z_{i}\right)=\gamma_{i}, \quad \varphi\left(u_{i}\right)=\delta_{i}
$$

$1 \leqslant i \leqslant \tilde{\theta}$ 
Proof. - (a) We have to show that $\gamma_{i}$ preserves the relations (5.8), (1.4), (1.5), (1.6), (5.9). This is clear for (5.8), (1.4), (1.5), (5.9). We check (1.6): let $\widetilde{\theta}+1 \leqslant j, h \leqslant \theta$ such that $\lambda_{j h} \neq 0$. Then

$$
\chi_{i}\left(g_{j} g_{h}\right)=\chi_{j} \chi_{h}\left(g_{i}\right)^{-1}=1,
$$

by (5.2) and (5.4). So that relations (1.6) hold and (a) is proven.

(b) This is equivalent to: there exists an algebra map $T: \mathcal{B} \rightarrow M_{2}(k)$ such that

$$
T\left(y_{k}\right)=\left(\begin{array}{cc}
1 & 0 \\
0 & \gamma_{i}\left(y_{k}\right)
\end{array}\right), \quad T\left(b_{j}\right)=\left(\begin{array}{cc}
0 & \tilde{\lambda}_{i j} \\
0 & 0
\end{array}\right),
$$

$1 \leqslant k \leqslant s, \tilde{\theta}+1 \leqslant j \leqslant \theta$. Then $T$ is of the form

$$
T(a)=\left(\begin{array}{cc}
\epsilon(a) & \delta_{i}(a) \\
0 & \gamma_{i}(a)
\end{array}\right)
$$

and $\delta_{i}$ is the desired derivation. So, we need to show that the relations (5.8), (1.4), (1.5), (1.6), (5.9) hold for the matrices in (5.23). This is evident for (5.8). For (1.4) it amounts to $\tilde{\lambda}_{i j} \chi_{i}\left(y_{k}\right)^{-1}=\tilde{\lambda}_{i j} \chi_{j}\left(y_{k}\right)$, which follows from (5.4) when $\tilde{\lambda}_{i j} \neq 0$. For (1.5) and (5.9) the argument is clear. Finally, the left hand side of (1.6) for $j<h$, is 0 , whereas the right-hand side also vanishes since $\lambda_{j h} \gamma_{i}\left(g_{j}\right) \gamma_{i}\left(g_{h}\right)=\lambda_{j h} \chi_{j}\left(g_{i}\right)^{-1} \chi_{h}\left(g_{i}\right)^{-1}=\lambda_{j h}$ by (5.4) again.

(c) It is enough to verify that $\delta_{i}, \gamma_{i}$ satisfy the defining relations (5.8), (1.4), (1.5), (1.6), (5.9) for $\mathcal{U}$. Indeed, this will automatically imply that $\varphi$ is a Hopf algebra map. Note that (1.6) are empty since the Dynkin diagram of $\mathcal{U}$ is connected. For (5.8), it is enough to verify that the equalities hold when applied to the generators $b_{\tilde{\theta}+1}, \ldots, b_{\theta}, y_{1}, \ldots, y_{s}$ since both sides are algebra maps. This is now not difficult; for instance

$$
\left(\gamma_{m} \gamma_{h}\right)\left(b_{j}\right)=\gamma_{m}\left(g_{j}\right) \gamma_{h}\left(b_{j}\right)+\gamma_{m}\left(b_{j}\right)=0=\left(\gamma_{h} \gamma_{m}\right)\left(b_{j}\right)
$$

The first relations in (5.8) for $\Upsilon$ hold since ord $\gamma_{i}$ divides ord $Z_{i}$ for all $i$. For (1.4) we need again to verify only on generators, since both sides are skew-derivations; this verification is in turn straightforward. The left-hand side of the Serre relations (1.5) is a skew-derivation by [5, Lemma A.1]; again we are reduced to see that $\left(\operatorname{ad} \delta_{i}\right)^{1-a_{i j}} \delta_{j}\left(b_{h}\right)=0=\left(\operatorname{ad} \delta_{i}\right)^{1-a_{i j}} \delta_{j}\left(y_{t}\right)$, $\tilde{\theta}+1 \leqslant h \leqslant \theta, 1 \leqslant t \leqslant s$. Write $\left(\operatorname{ad} \delta_{i}\right)^{1-a_{i j}} \delta_{j}=\delta_{i}\left(\operatorname{ad} \delta_{i}\right)^{-a_{i j}} \delta_{j}-\hat{q}\left(\left(\operatorname{ad} \delta_{i}\right)^{-a_{i j}} \delta_{j}\right) \delta_{i}$, where $\hat{q}$ is a root of 1 . Then

$$
\left(\operatorname{ad} \delta_{i}\right)^{1-a_{i j}} \delta_{j}\left(y_{t}\right)=\delta_{i}\left(y_{t}\right)\left(\operatorname{ad} \delta_{i}\right)^{-a_{i j}} \delta_{j}\left(y_{t}\right)-\hat{q}\left(\operatorname{ad} \delta_{i}\right)^{-a_{i j}} \delta_{j}\left(y_{t}\right) \delta_{i}\left(y_{t}\right)=0 .
$$

Similarly,

$$
\left(\operatorname{ad} \delta_{i}\right)^{1-a_{i j}} \delta_{j}\left(b_{h}\right)=\delta_{i}\left(b_{h}\right)\left(\operatorname{ad} \delta_{i}\right)^{-a_{i j}} \delta_{j}(1)-\hat{q}\left(\operatorname{ad} \delta_{i}\right)^{-a_{i j}} \delta_{j}\left(g_{h}\right) \delta_{i}\left(b_{h}\right)=0,
$$

since $\left(\operatorname{ad} \delta_{i}\right)^{-a_{i j}} \delta_{j}$ is a homogeneous polynomial in $\delta_{i}, \delta_{j}$ of positive degree. Finally, relations (5.9) follow from the next lemma.

LEMMA 5.24. - Let $B$ be a finite dimensional pointed Hopf algebra generated as an algebra by group-like elements and a family $b_{j}, j \in \mathcal{J}$, of $\left(h_{j}, 1\right)$-primitives, for some $h_{j} \in G(B)$. Let $\widehat{\mathcal{U}}$ be the algebra presented by generators $u_{1}, \ldots, u_{\tilde{\theta}}$ and $z_{1}, \ldots, z_{\tilde{\theta}}$ with exactly the same relations 
as for $\mathcal{U}$ except for (5.9); it is a Hopf algebra via (1.9). Let $N=N_{J}$. Assume there exists a Hopf algebra map $\phi: \widehat{\mathcal{U}} \rightarrow\left(B^{*}\right)^{\mathrm{cop}}$ such that $\gamma_{i}:=\phi\left(z_{i}\right)$ and $\delta_{i}:=\phi\left(u_{i}\right)$ satisfy

$$
\gamma_{i}\left(b_{j}\right)=0, \quad \delta_{i}(g)=0, \quad j \in \mathcal{J}, g \in G(B)
$$

for all $1 \leqslant i \leqslant \tilde{\theta}$. Then $\phi\left(u_{\alpha}^{N}\right)=0$ for all $\alpha \in \Phi_{J}^{+}$.

Proof. - There exists a Hopf algebra projection $\varpi: \widehat{\mathcal{U}} \rightarrow \mathbb{k} \Upsilon$ such that $\varpi\left(u_{i}\right)=0$ and $\varpi\left(z_{i}\right)=$ $z_{i}$ for all $i$. Let $\mathcal{K}$ be the subalgebra of $\widehat{\mathcal{U}}$ generated by $u_{\alpha}^{N}, \alpha \in \Phi_{J}^{+}$, and $z_{i}^{N}, 1 \leqslant i \leqslant \tilde{\theta}$. We claim that

$$
\phi(u)=\phi(\varpi(u))
$$

for all $u \in \mathcal{K}$. Clearly, this implies the lemma. By Theorem 4.8, we know that $\mathcal{K}$ is a Hopf subalgebra of $\widehat{\mathcal{U}}$. We have to prove that $\phi(u)(b)=\phi(\varpi(u))(b)$ for $b$ a monomial in the grouplikes of $B$ and the $b_{j}$ 's. We do this by induction on the length of the monomial.

We first check the case of length 1 . Here we show more generally that

$$
\phi(u)(g)=\phi(\varpi(u))(g) \quad \text { and } \quad \phi(u)\left(b_{j}\right)=\phi(\varpi(u))\left(b_{j}\right)
$$

for all $g \in G(B), j \in \mathcal{J}$ and $u \in G(\widehat{\mathcal{U}})$ or $u$ of the form $u_{i_{1}} \cdots u_{i_{t}} z$, with $z$ group-like, $1 \leqslant i_{1}, \ldots, i_{t} \leqslant \tilde{\theta}$, and $t \geqslant 2$. Note that each element in $\mathcal{K}$ is a linear combination of such $u$ 's since $N \geqslant 2$. The case when $u$ is a group-like is clear. Let $u=u_{i_{1}} \cdots u_{i_{t}} z$, with $z$ group-like, $1 \leqslant i_{1}, \ldots, i_{t} \leqslant \tilde{\theta}$, and $t \geqslant 2$. Then $\phi(u)=\delta_{i_{1}} \cdots \delta_{i_{t}} \phi(z)$, and

$$
\begin{aligned}
\phi(u)(g) & =\delta_{i_{1}}(g) \cdots \delta_{i_{t}}(g) \phi(z)(g)=0, \\
\phi(u)\left(b_{j}\right) & =\sum_{1 \leqslant r \leqslant t+1} \delta_{i_{1}}\left(g_{j}\right) \cdots \delta_{i_{r-1}}\left(g_{j}\right) \delta_{i_{r}}\left(b_{j}\right) \delta_{i_{r+1}}(1) \cdots \phi(z)(1)=0,
\end{aligned}
$$

where we used (5.25) and $t \geqslant 2$.

Assume then that $b=c d$ where $c$ and $d$ are monomials satisfying the claim. Since $\phi$ and $\varpi$ are Hopf algebra maps, we have

$$
\phi(u)(c d)=\phi\left(u_{(2)}\right)(c) \phi\left(u_{(1)}\right)(d)=\phi\left(\varpi\left(u_{(2)}\right)\right)(c) \phi\left(\varpi\left(u_{(1)}\right)\right)(d)=\phi(\varpi(u))(c d) .
$$

We are ready now to conclude the proof of the theorem. Consider the cocycle

$$
\sigma:(\mathcal{U} \otimes \mathcal{B}) \otimes(\mathcal{U} \otimes \mathcal{B}) \rightarrow \mathbb{k}
$$

obtained as in Lemma 5.14 from the map $\varphi$ constructed in Lemma 5.19. Consider the Hopf algebra $(\mathcal{U} \otimes \mathcal{B})_{\sigma}$; it has dimension $|\Upsilon||\Gamma| \prod_{I \in \mathcal{X}} N_{I}^{\left|\Phi_{I}^{+}\right|}$. We claim that the group-like elements $z_{i} \otimes g_{i}^{-1}$ are central in $(\mathcal{U} \otimes \mathcal{B})_{\sigma}$ for all $i$. By definition of $(\mathcal{U} \otimes \mathcal{B})_{\sigma}$, we have to show for all $u \in \mathcal{U}, b \in \mathcal{B}$ and $1 \leqslant i \leqslant \tilde{\theta}$

$$
\varphi\left(z_{i}\right)\left(b_{(1)}\right) u z_{i} \otimes b_{(2)} g_{i}^{-1} \varphi\left(z_{i}^{-1}\right)\left(b_{(3)}\right)=\varphi\left(u_{(1)}\right)\left(g_{i}^{-1}\right) z_{i} u_{(2)} \otimes g_{i}^{-1} b \varphi\left(\mathcal{S} u_{(3)}\right)\left(g_{i}^{-1}\right) .
$$

Since $u \otimes b=(u \otimes 1)(1 \otimes b)$ in $(\mathcal{U} \otimes \mathcal{B})_{\sigma}$ for all $u \in \mathcal{U}, b \in \mathcal{B}$, it is enough to check (5.27) on generators of $\mathcal{U}$ and $\mathcal{B}$. This in turn follows easily from the definitions.

Let $\widetilde{\mathcal{A}}$ be the quotient of $(\mathcal{U} \otimes \mathcal{B})_{\sigma}$ by the central Hopf subalgebra $\mathbb{k}\left[z_{i} \otimes g_{i}^{-1}: 1 \leqslant i \leqslant \tilde{\theta}\right]$ with quotient map $\pi$. Then $\operatorname{dim} \widetilde{\mathcal{A}}=|\Gamma| \prod_{I \in \mathcal{X}} N_{I}^{\left|\Phi_{I}^{+}\right|}$by a result of the second author [26, Th. 3.3.1]. 
Next we claim the existence of a surjective algebra map $\mathcal{F}: \mathcal{A} \rightarrow \widetilde{\mathcal{A}}$ such that

$$
\mathcal{F}\left(a_{i}\right)=\pi\left(u_{i} \otimes 1\right), \quad \mathcal{F}\left(a_{j}\right)=\pi\left(1 \otimes b_{j}\right), \quad \mathcal{F}\left(y_{k}\right)=\pi\left(1 \otimes y_{k}\right),
$$

for $1 \leqslant i \leqslant \tilde{\theta}, \tilde{\theta}+1 \leqslant j \leqslant \theta, 1 \leqslant k \leqslant s$. Again we have to verify the relations (5.8), (1.4), (1.5), (1.6), (5.9). Up to (1.6) these relations already hold in $(\mathcal{U} \otimes \mathcal{B})_{\sigma}$. For (1.6), it is enough to show that

$$
\begin{aligned}
& \pi\left(u_{i} \otimes 1\right) \pi\left(1 \otimes b_{j}\right)-\chi_{j}\left(g_{i}\right) \pi\left(1 \otimes b_{j}\right) \pi\left(u_{i} \otimes 1\right)=\lambda_{i j}\left(1-\pi\left(1 \otimes g_{i} g_{j}\right)\right), \\
& 1 \leqslant i \leqslant \tilde{\theta}, \tilde{\theta}+1 \leqslant j \leqslant \theta .
\end{aligned}
$$

A tedious computation shows that the left-hand side is equal to $\chi_{j}\left(g_{i}\right) \tilde{\lambda}_{i j}\left(\pi\left(z_{i} \otimes g_{j}\right)-1\right)$. Since $\pi\left(z_{i} \otimes g_{i}{ }^{-1}\right)=1$, we have $\pi\left(z_{i} \otimes g_{j}\right)=\pi\left(1 \otimes g_{i} g_{j}\right)$. Hence the claim follows if we choose $\tilde{\lambda}_{i j}=-\chi_{i}\left(g_{j}\right) \lambda_{i j}$ for all $1 \leqslant i \leqslant \tilde{\theta}, \tilde{\theta}+1 \leqslant j \leqslant \theta$.

On the other hand, we have algebra maps $\mathcal{G}_{1}: \mathcal{U} \rightarrow \mathcal{A}, \mathcal{G}_{2}: \mathcal{B} \rightarrow \mathcal{A}$ given by $\mathcal{G}_{1}\left(u_{i}\right)=a_{i}$, $\mathcal{G}_{1}\left(z_{i}\right)=g_{i}, \mathcal{G}_{2}\left(b_{j}\right)=a_{j} \mathcal{G}_{2}\left(y_{k}\right)=y_{k}, 1 \leqslant i \leqslant \tilde{\theta}, \tilde{\theta}+1 \leqslant j \leqslant \theta, 1 \leqslant k \leqslant s$. Here we use that ord $g_{i}$ divides ord $Z_{i}$ for all $i$. Let $\mathcal{G}: \mathcal{U} \otimes \mathcal{B} \rightarrow \mathcal{A}$ be defined by $\mathcal{G}(u \otimes b)=\mathcal{G}_{1}(u) \mathcal{G}_{2}(b)$ for all $u \in \mathcal{U}, b \in \mathcal{B}$. We claim that $\mathcal{G}$ is an algebra map. By Lemma 5.15, we have to verify

$$
\varphi\left(u_{(1)}\right)\left(b_{(1)}\right) \mathcal{G}_{1}\left(u_{(2)}\right) \mathcal{G}_{2}\left(b_{(2)}\right)=\mathcal{G}_{2}\left(b_{(1)}\right) \mathcal{G}_{1}\left(u_{(1)}\right) \varphi\left(u_{(2)}\right)\left(b_{(2)}\right),
$$

for all generators. This is a straightforward task; for the case $u_{i}$ and $b_{j}$ we need again the condition $\tilde{\lambda}_{i j}=-\chi_{i}\left(g_{j}\right) \lambda_{i j}$.

Since clearly $\mathcal{G}$ factorizes through $\widetilde{\mathcal{A}}, \mathcal{F}$ is an isomorphism and the theorem follows.

\section{Lifting of relations}

In this section, we assume the situation described in Section 4. To lift the Serre relations, we need the following lemma.

LEMMA 6.1. - Let $1 \leqslant i \neq j \leqslant \theta$ and let I be the connected component containing $i$.

(a) If $i \sim j$, assume that $N_{I} \neq 3$; if $i \sim j$ and I is of type $B_{n}, C_{n}$ or $F_{4}$ assume further $N_{I} \neq 5$. Then there exists no $\ell, 1 \leqslant \ell \leqslant \theta$, such that $g_{i}^{1-a_{i j}} g_{j}=g_{\ell}, \chi_{i}^{1-a_{i j}} \chi_{j}=\chi_{\ell}$.

(b) Assume that $i \sim j$ and $N_{I} \neq 3$. If I is of type $B_{n}, C_{n}$ or $F_{4}$, resp. $G_{2}$, assume further that $N_{I} \neq 5$, resp. $N_{I} \neq 7$. Then $\chi_{i}^{1-a_{i j}} \chi_{j} \neq \varepsilon$.

Proof. - (a) Assume that $g_{i}^{1-a_{i j}} g_{j}=g_{\ell}, \chi_{i}^{1-a_{i j}} \chi_{j}=\chi_{\ell}$ for some $\ell$. Substituting $g_{\ell}$ and $\chi_{\ell}$ in $\left\langle\chi_{\ell}, g_{i}\right\rangle\left\langle\chi_{i}, g_{\ell}\right\rangle=q_{i}^{a_{i \ell}}$ and using $\left\langle\chi_{j}, g_{i}\right\rangle\left\langle\chi_{i}, g_{j}\right\rangle=q_{i}^{a_{i j}}$ we conclude that

$$
N_{i} \text { divides } 2-a_{i j}-a_{i \ell} \text {. }
$$

Changing the rôles of $i$ and $j$ we obtain in the same way

$$
N_{j} \text { divides } a_{j i}\left(1-a_{i j}\right)-a_{j \ell}+2 \text {. }
$$

First assume that $i \nsim j$. In particular, $a_{j i}=0=a_{i j}$ and $a_{i \ell}=0$ or $a_{j \ell}=0$. If $a_{i \ell}=0$, resp. $a_{j \ell}=0$, then we get from (6.2), resp. (6.3), that $N_{i}=2$, resp. $N_{j}=2$, which is not possible.

Next assume that $i \sim j$. If $j=\ell$ then $N_{i}$ divides $2\left(1-a_{i j}\right)$ by (6.2). The only possibility is $a_{i j}=-2$ and $N_{i}=3$; but this was excluded in the hypothesis. If $i=\ell$ then $N_{i}$ divides $-a_{i j}$ 
by (6.2) and $N_{j}$ divides $a_{j i} a_{i j}-2$ by (6.3); but this contradicts our general assumptions on the $N_{i}$ 's.

Finally, if $i \neq \ell$ and $j \neq \ell$ then $a_{i \ell} \neq-3$ and $a_{j \ell} \neq-3$. We discuss the different possible values of $a_{i j}$. If $a_{i j}=0$ or -1 , by (6.2) and since $N_{i}$ is odd we see that $N_{i}=3$ or 5 , cases excluded by hypothesis. If $a_{i j}=-2$ then $a_{j i}=-1$. By (6.3), $N_{j}$ divides $-1-a_{j \ell}$; this discards everything except $a_{j \ell}=-1$. But in this last case, $a_{i \ell}=0$ and $N_{i}$ divides 4 by (6.2), a contradiction. Finally, $a_{i j}=-3$ is impossible by analogous arguments.

(b) Assume that $\chi_{i}^{1-a_{i j}} \chi_{j}=\varepsilon$. We consider first the case $a_{i j} \neq 0$. Evaluating at $g_{i}$, we get $q_{i}^{1-a_{i j}} \chi_{j}\left(g_{i}\right)=1$; hence $q_{i}=\chi_{i}\left(g_{j}\right)$. Evaluating at $g_{j}$, we get then $q_{j}=q_{i}^{a_{i j}-1}$. Since $q_{j}^{a_{j i}}=q_{i}^{a_{i j}}$ we finally obtain

$$
N_{i} \text { divides } a_{i j} a_{j i}-a_{i j}-a_{j i} \text {. }
$$

The possible values of $a_{i j} a_{j i}-a_{i j}-a_{j i}$ are 3,5 or 7 , where 5 , resp. 7 , is only possible if $I$ is of type $B_{n}, C_{n}$ or $F_{4}$, resp. $G_{2}$. This contradicts the hypothesis.

We consider finally the case $a_{i j}=0$; so that $\chi_{i} \chi_{j}=\varepsilon$. Since $I$ is connected, there is a sequence $i=i_{1}, i_{2}, \ldots, i_{t}=j$ of elements in $I$ such that $a_{i_{\ell} i_{\ell+1}} \neq 0,2$ for all $\ell, 1 \leqslant \ell<t$. Then

$$
q_{i}^{a_{i_{1} i_{2}} a_{i_{2} i_{3} \ldots a_{i_{t-1} i_{t}}}}=q_{i_{2}}^{a_{i_{2} i_{1}} a_{i_{2} i_{3} \ldots a_{i_{t-1} i_{t}}}}=\cdots=q_{j}^{a_{i_{2} i_{1}} a_{i_{3} i_{2}} \ldots a_{i_{t}} i_{t-1}},
$$

by substituting $q_{i}^{a_{i_{1} i_{2}}}=q_{i_{2}}^{a_{i_{2} i_{1}}}$, then $q_{i_{2}}^{a_{i_{2} i_{1}}}=q_{i_{3}}^{a_{i_{3} i_{2}}}$ and so on. Note that $q_{i}=q_{j}^{-1}$ since $a_{i j}=0$ and $\chi_{i} \chi_{j}=\varepsilon$. Hence

$$
N_{i} \text { divides } a_{i_{1} i_{2}} a_{i_{2} i_{3}} \ldots a_{i_{t-1} i_{t}}+a_{i_{2} i_{1}} a_{i_{3} i_{2}} \ldots a_{i_{t} i_{t-1}} .
$$

The possible values of the sum in (6.5) are \pm 2 or \pm 3 . Hence (6.5) contradicts our assumptions in (b).

Let now $A$ be a pointed Hopf algebra with $G(A) \simeq \Gamma$, not necessarily finite dimensional. Let $R$ be the diagram of $A$ (see Section 2.1). We assume there is an isomorphism $\mathcal{P}(R) \cong V$ in ${ }_{\Gamma}^{\Gamma} \mathcal{Y} \mathcal{D}$. Then

$$
\bigoplus_{\substack{g, h \in \Gamma \\ \varepsilon \neq \chi \in \widehat{\Gamma}}} \mathcal{P}_{g, h}(A)^{\chi} \cong A_{1} / A_{0} \cong V \# \mathbb{k} \Gamma
$$

(see [4, Lemma 5.4]). Let $a_{i} \in \mathcal{P}_{g_{i}, 1}(A)^{\chi_{i}}, 1 \leqslant i \leqslant \theta$, such that $\overline{a_{i}}$ is mapped onto $x_{i}$ for all $i$. Then we know from [5, Lemma 5.4] that for all $g \in \Gamma, \chi \in \widehat{\Gamma}$ with $\chi \neq \varepsilon$ :

$$
\begin{aligned}
& \mathcal{P}_{g, 1}(A)^{\chi} \neq 0 \Longleftrightarrow \text { there is some } 1 \leqslant \ell \leqslant \theta: g=g_{\ell}, \chi=\chi_{\ell} ; \\
& \mathcal{P}_{g, 1}(A)^{\varepsilon}=\mathbb{k}(1-g) .
\end{aligned}
$$

Recall that we have fixed $\Gamma,\left(a_{i j}\right)_{1 \leqslant i, j \leqslant \theta},\left(g_{i}\right)_{1 \leqslant i \leqslant \theta},\left(\chi_{j}\right)_{1 \leqslant j \leqslant \theta}$ as in the situation of Section 4.

THEOREM 6.8. - Let $A$ and $a_{1}, \ldots, a_{\theta}$ be as above.

(a) There is a linking datum $\mathcal{D}\left(\Gamma,\left(a_{i j}\right)_{1 \leqslant i, j \leqslant \theta},\left(g_{i}\right)_{1 \leqslant i \leqslant \theta},\left(\chi_{j}\right)_{1 \leqslant j \leqslant \theta},\left(\lambda_{i j}\right)_{1 \leqslant i<j \leqslant \theta, i \nsim j}\right)$ with linking elements $\left(\lambda_{i j}\right)_{1 \leqslant i<j \leqslant \theta, i \nsim j}$ such that (1.6) holds.

(b) Let $I \in \mathcal{X}$. Assume that $N_{I} \neq 3$. If I is of type $B_{n}, C_{n}$ or $F_{4}$, resp. $G_{2}$, assume further that $N_{I} \neq 5$, resp. $N_{I} \neq 7$. Then the quantum Serre relations (1.5) hold for all $i \neq j \in I$. 
Proof. - It is known that $\left(\operatorname{ad} a_{i}\right)^{1-a_{i j}} a_{j} \in \mathcal{P}_{g_{i}^{1-a_{i j}} g_{j}, 1}(A)^{\chi_{i}^{1-a_{i j}} \chi_{j}}$, see for instance [5, Appendix]. Part (b) of the theorem then follows from Lemma 6.1, (6.6) and (6.7).

To prove part (a), let us assume that $i \nsim j$. By Lemma 6.1, (6.6) and (6.7) again, $a_{i} a_{j}-$ $\chi_{j}\left(g_{i}\right) a_{j} a_{i}=\lambda_{i j}\left(1-g_{i} g_{j}\right)$, for some $\lambda_{i j} \in \mathbb{k}$. We can choose $\lambda_{i j}=0$ when $g_{i} g_{j}=1$ or else if $\chi_{i} \chi_{j} \neq \varepsilon$. That is, $\lambda_{i j}$ is a linking datum for $\left(a_{i j}\right), g_{1}, \ldots, g_{\theta}$ and $\chi_{1}, \ldots, \chi_{\theta}$; and (1.6) holds.

LEMMA 6.9. - Let $A$ and $a_{1}, \ldots, a_{\theta}$ be as above. Assume further that

- the hypothesis from Theorem 6.8 part (b) holds for all $I \in \mathcal{X}$.

- A is finite dimensional.

- $g_{i}^{N_{i}}=1,1 \leqslant i \leqslant \theta$.

Then the relations (5.9) hold in $A$.

Proof. - Let us fix $I \in \mathcal{X}$. Let $\widehat{\mathcal{U}}$ be the algebra presented by generators $\hat{a}_{i}, i \in I, y_{1}, \ldots, y_{s}$ and relations (5.8), (1.4), (1.5) and (1.6); it is a Hopf algebra via (1.9). Let $N=N_{I}$ and let $\mathcal{K}$ be the subalgebra of $\widehat{\mathcal{U}}$ generated by $\hat{a}_{\alpha}^{N}, \alpha \in \Phi_{I}^{+}$, and $g_{i}^{N}, i \in I$. By Theorem 4.8, we know that $\mathcal{K}$ is a Hopf subalgebra of $\widehat{\mathcal{U}}$. Note that $\mathcal{K}$ is a graded Hopf algebra with trivial coradical. By the choice of the $a_{i}$ 's in $A$ and Theorem 6.8, we see there is a well-defined Hopf algebra map $\widehat{\mathcal{U}} \rightarrow A$ such that $\hat{a}_{i} \mapsto a_{i}, i \in I$. The image of $\mathcal{K}$ under this map is a finite dimensional pointed Hopf algebra; it has a trivial coradical by [26] and therefore it is trivial. This implies the lemma.

THEOREM 6.10. - Let $A$ be as above and assume that

- the hypothesis from Theorem 6.8 part (b) holds for all $I \in \mathcal{X}$.

- $\operatorname{gr} A \simeq \mathfrak{B}(V) \# \mathbb{k} \Gamma$, hence $A$ is finite dimensional.

- $g_{i}^{N_{i}}=1,1 \leqslant i \leqslant \theta$.

Then there exists a linking datum

$$
\mathcal{D}=\mathcal{D}\left(\Gamma,\left(a_{i j}\right)_{1 \leqslant i, j \leqslant \theta},\left(g_{i}\right)_{1 \leqslant i \leqslant \theta},\left(\chi_{j}\right)_{1 \leqslant j \leqslant \theta},\left(\lambda_{i j}\right)_{1 \leqslant i<j \leqslant \theta, i \nsim j}\right)
$$

such that

$$
A \simeq \mathfrak{u}(\mathcal{D}) .
$$

Proof. - By Theorem 6.8 and Lemma 6.9, there exist elements $\left(\lambda_{i j}\right)_{1 \leqslant i<j \leqslant \theta, i \nsim j}$ such that

$$
\mathcal{D}=\mathcal{D}\left(\Gamma,\left(a_{i j}\right)_{1 \leqslant i, j \leqslant \theta},\left(g_{i}\right)_{1 \leqslant i \leqslant \theta},\left(\chi_{j}\right)_{1 \leqslant j \leqslant \theta},\left(\lambda_{i j}\right)_{1 \leqslant i<j \leqslant \theta, i \nsim j}\right)
$$

is a linking datum, and a surjective Hopf algebra map $\mathcal{J}: \mathcal{A} \rightarrow A$, where $\mathcal{A}=\mathfrak{u}(\mathcal{D})$. But $\operatorname{dim} A=\operatorname{dim} \operatorname{gr} A=|\Gamma| \operatorname{dim} \mathfrak{B}(V)=\operatorname{dim} \mathcal{A}$ by Theorems 4.5 and 5.17 ; hence $\mathcal{J}$ is an isomorphism.

\section{Hopf algebras generated in degree one}

In this section, $\Gamma$ is a finite abelian group. Let $V \in_{\Gamma}^{\Gamma} \mathcal{Y} \mathcal{D}$ be of Cartan type with braiding $\left(b_{i j}\right)$ and Cartan matrix $\left(a_{i j}\right)$. As in [5] we say that the braiding $\left(b_{i j}\right)$ satisfies the relative primeness condition if for all $i, j,\left(a_{i j}\right)$ is 0 or relatively prime to the order of $b_{i i}$.

The next lemma follows from [5]. We will apply it in the case of $2 \times 2$ (hence symmetrizable) Cartan matrices.

Lemma 7.1. - Let $V \in \in_{\Gamma}^{\Gamma} \mathcal{Y} \mathcal{D}$ be of symmetrizable Cartan type with braiding $\left(b_{i j}\right)_{1 \leqslant i, j \leqslant \theta}$. Assume that for all $1 \leqslant i, j \leqslant \theta$, the order of $b_{i j}$ is odd, and that $\left(b_{i j}\right)_{1 \leqslant i, j \leqslant \theta}$ satisfies the relative primeness condition. If $\mathfrak{B}(V)$ is finite-dimensional, then $V$ is of finite Cartan type. 
Proof. - By [5, Lemma 4.1] we can realize the braiding over a suitable finite abelian group $\widetilde{\Gamma}$ and twist with a 2 -cocycle $F$ such that the resulting braiding $\left(b_{i j}^{F}\right)$ is symmetric with elements of odd order, has the same diagonal elements and is of Cartan type with the same Cartan matrix $\left(a_{i j}\right)$ as $V$. We can now conclude from [5, Lemma 4.3] that $\left(b_{i j}^{F}\right)$ is of FL-type (see [5]). Let $\widetilde{V}^{F}$ be the Yetter-Drinfeld module over $\widetilde{\Gamma}$ with braiding $\left(b_{i j}^{F}\right)$. Since $\mathfrak{B}(V)$ and $\mathfrak{B}\left(\widetilde{V}^{F}\right)$ have the same dimension, $\mathfrak{B}\left(\widetilde{V}^{F}\right)$ is finite-dimensional. Then $\left(a_{i j}\right)$ is of finite Cartan type by [5, Theorem 3.1].

LEMmA 7.2. - Let $S=\bigoplus_{n \geqslant 0} S(n)$ be a finite-dimensional graded Hopf algebra in ${ }_{\Gamma}^{\Gamma} \mathcal{Y D}$ such that $S(0)=\mathbb{k} 1$. Assume that $S(1)$ is of finite Cartan type with basis $\left(x_{i}\right)_{1 \leqslant i \leqslant \theta}$, braiding $\left(b_{i j}\right)_{1 \leqslant i, j \leqslant \theta}$ and Cartan matrix $\left(a_{i j}\right)_{1 \leqslant i, j \leqslant \theta}$ as in (3.7). For all $1 \leqslant l \leqslant \theta$, let $q_{l}=b_{l l}$ and $N_{l}=\operatorname{ord}\left(q_{l}\right)$.

Let $1 \leqslant i, j \leqslant \theta, i \neq j$, and assume that $N_{i}, N_{j}$ and $\operatorname{ord}\left(b_{i j}\right)$ are odd, and $N_{i}$ is not divisible by 3 and $>7$.

(a) Assume $i \sim j$ and let $I$ be the connected component containing $i, j$. If the type of $I$ is $B_{n}, C_{n}$ or $F_{4}$, assume that $N_{i}$ is not divisible by 5 . If the type is $G_{2}$, assume that $N_{i}$ is not divisible by 5 or 7 . Then $\left(\operatorname{ad}_{c} x_{i}\right)^{1-a_{i j}} x_{j}=0$.

(b) Assume $i \nsim j$ and $q_{i} q_{j}=1$ or $\operatorname{ord}\left(q_{i} q_{j}\right)=\operatorname{ord}\left(q_{i}\right)$. Then $x_{i} x_{j}-b_{i j} x_{j} x_{i}=0$.

Proof. - Define $z_{1}:=x_{i}, z_{2}:=\left(\operatorname{ad}_{c} x_{i}\right)^{1-a_{i j}} x_{j}$. In both cases we have to show $z_{2}=0$. We assume that $z_{2}$ is not 0 . Let $g_{i} \in \Gamma, \chi_{i} \in \widehat{\Gamma}, 1 \leqslant i, j \leqslant \theta$, with $b_{i j}=\chi_{j}\left(g_{i}\right)$ for all $i, j$. Then action and coaction on $z_{1}, z_{2}$ are given by $\delta\left(z_{1}\right)=g_{i} \otimes z_{1}, \delta\left(z_{2}\right)=g_{i}^{1-a_{i j}} g_{j} \otimes z_{2}$ and $h \cdot z_{1}=\chi_{i}(h) z_{1}$, $h \cdot z_{2}=\left(\chi_{i}^{1-a_{i j}} \chi_{j}\right)(h) z_{2}$ for all $h \in \Gamma$. The elements $z_{1}, z_{2}$ are linearly independent since they are non-zero and of different degree. The braiding $\left(B_{k l}\right)_{1 \leqslant k, l \leqslant 2}$ of the 2-dimensional YetterDrinfeld module with basis $z_{1}, z_{2}$ is given by

$$
\begin{array}{ll}
B_{11}=\chi_{i}\left(g_{i}\right)=q_{i}, & B_{12}=\left(\chi_{i}^{1-a_{i j}} \chi_{j}\right)\left(g_{i}\right)=q_{i} b_{j i}^{-1}, \\
B_{21}=\chi_{i}\left(g_{i}^{1-a_{i j}} g_{j}\right)=q_{i}^{1-a_{i j}} b_{j i}, & B_{22}=\left(\chi_{i}^{1-a_{i j}} \chi_{j}\right)\left(g_{i}^{1-a_{i j}} g_{j}\right)=q_{i}^{1-a_{i j}} q_{j} .
\end{array}
$$

Then $B_{12} B_{21}=q_{i}^{2-a_{i j}}$. We claim that $\left(B_{k l}\right)$ is of Cartan type and satisfies the relative primeness condition, that is there are integers $A_{12}, A_{21}$ such that

$$
\begin{aligned}
& q_{i}^{2-a_{i j}}=q_{i}^{A_{12}} \text { and } A_{12} \text { is relatively prime to } N_{i}, \\
& q_{i}^{2-a_{i j}}=\left(q_{i}^{1-a_{i j}} q_{j}\right)^{A_{21}} \text {, and } A_{21} \text { is relatively prime to ord }\left(q_{i}^{1-a_{i j}} q_{j}\right) .
\end{aligned}
$$

In both cases $2-a_{i j}$ is relatively prime to $N_{i}$, because of the hypothesis on $N_{i}$. This shows (7.3).

We now prove (7.4) in case (a). Then $N_{I}=N_{i}=N_{j}$, and it suffices to find an integer $A_{21}$ relatively prime to $N_{i}$ with $q_{i}^{2-a_{i j}}=\left(q_{i}^{1-a_{i j}} q_{j}\right)^{A_{21}}$.

First assume that $a_{i j} \neq 0$. Since $a_{j i} \neq 0$ is relatively prime to $N_{i}$, it is enough to consider the $a_{j i}$ th power of (7.4). Since $q_{i}^{a_{i j}}=q_{j}^{a_{j i}}$ by the Cartan condition for $\left(b_{i j}\right)$, we have to solve $\left(2-a_{i j}\right) a_{j i} \equiv\left(\left(1-a_{i j}\right) a_{j i}+a_{i j}\right) A_{21} \bmod N_{i}$. Since $\left(a_{i j}\right)$ is of finite Cartan type, the possible values of $\left(2-a_{i j}\right) a_{j i}$ are $-3,-4,-5,-6,-9(-4,-6$ resp. $-5,-9$ only occur if the type is $B_{n}, C_{n}$ or $F_{4}$ resp. $\left.G_{2}\right)$; the possible values of $\left(\left(1-a_{i j}\right) a_{j i}+a_{i j}\right)$ are $-3,-5,-7,(-5$, resp. -7 only occur if the type is $B_{n}, C_{n}$ or $F_{4}$ resp. $\left.G_{2}\right)$. Hence $\left(2-a_{i j}\right) a_{j i}$ and $\left(\left(1-a_{i j}\right) a_{j i}+a_{i j}\right)$ are relatively prime to $N_{i}$ by assumption, and the claim follows. (Note that $a+b$ is never 0 .) 
If $a_{i j}=0$, then by connectedness there is a sequence $i=i_{1}, i_{2}, \ldots, i_{t}=j$ of elements in $I$ such that $a_{i_{\ell} i_{\ell+1}} \neq 0,2$ for all $\ell, 1 \leqslant \ell<t$. Then as in the proof of Lemma 6.1(b),

$$
q_{i}^{a}=q_{j}^{b}, \quad \text { where } a=a_{i_{1} i_{2}} a_{i_{2} i_{3}} \ldots a_{i_{t-1} i_{t}}, \text { and } b=a_{i_{2} i_{1}} a_{i_{2} i_{3}} \ldots a_{i_{t-1} i_{t}} .
$$

Since the possible values of $a, b$ are $1,2,-1,-2$, the $b$ th power of (7.4) leads to the congruence $2 b \equiv(b+a) A_{21} \bmod N_{i}$, and the claim again follows.

Assume case (b), in particular $a_{i j}=0$. If $q_{i} q_{j}=1$, we get a contradiction since the algebra generated by $z_{1}, z_{2}$ is finite-dimensional, hence $B_{22} \neq 1$ by [4, Lemma 3.1]. If $\operatorname{ord}\left(q_{i} q_{j}\right)=\operatorname{ord}\left(q_{i}\right),(7.4)$ is solvable since $N_{i}$ is odd.

Thus we have shown that $\left(B_{k l}\right)$ is of Cartan type and satisfies the relative primeness condition. Hence $\left(B_{k l}\right)$ is of finite Cartan type by Lemma 7.1. In both cases $A_{12}=2-a_{i j}-N_{i}$ is a solution of (7.3), and $-N_{i}<A_{12} \leqslant 0$. Hence the possible values of $A_{12}$ are $0,-1,-2$, -3 , and we see that $N_{i} \leqslant 8$. This contradicts our assumption, and we have shown the Serre relation $z_{2}=0$.

LEMma 7.5. - Let $S=\bigoplus_{n \geqslant 0} S(n)$ be a finite-dimensional graded Hopf algebra in ${ }_{\Gamma}^{\Gamma} \mathcal{Y D}$ such that $S(0)=\mathbb{k} 1$. Assume that $V=S(1)$ is of Cartan type with basis $\left(x_{i}\right)_{1 \leqslant i, j \leqslant \theta}$ as described in the beginning of Section 4. Assume the Serre relations

$$
\left(\operatorname{ad}_{c} x_{i}\right)^{1-a_{i j}} x_{j}=0 \quad \text { for all } 1 \leqslant i, j \leqslant \theta, i \neq j \text { and } i \sim j .
$$

Then the root vector relations

$$
x_{\alpha}^{N_{I}}=0, \quad \text { for all } \alpha \in \Phi_{I}^{+}, I \in \mathcal{X},
$$

hold in $S$.

Proof. - We fix a connected component $I \in \mathcal{X}$. Let $V_{I}$ be the Yetter-Drinfeld submodule of $V$ with basis $x_{i}, i \in I$, and $\widehat{\mathfrak{B}}\left(V_{I}\right)$ the quotient of $T\left(V_{I}\right)$ modulo the Serre relations of all elements $x_{i}, x_{j}$ with $i \neq j$ in I. Let $N_{I}=N$. The map $\Psi: T\left(V_{I}\right) \subset T(V) \rightarrow S$ factorizes over $\widehat{\mathfrak{B}}\left(V_{I}\right)$, since the Serre relations hold in $S$. By Theorem 4.8 the subalgebra $\mathcal{K}\left(V_{I}\right)$ of $\widehat{\mathfrak{B}}(V)$ generated by the powers of the root vectors $x_{\alpha}^{N_{I}}, \alpha \in \Phi_{I}^{+}$, is a braided Hopf subalgebra. As a coalgebra, $\mathcal{K}\left(V_{I}\right)$ is pointed and has trivial coradical. Hence $K:=\Psi\left(\mathcal{K}\left(V_{I}\right)\right)$ is a finitedimensional pointed and graded Hopf subalgebra of $S$ in ${ }_{\Gamma}^{\Gamma} \mathcal{Y} \mathcal{D}$ with trivial coradical. We have to show the root vector relation $x_{\alpha}^{N_{I}}=0, \alpha \in \Phi_{I}^{+}$in $S$, or equivalently that $K$ is one-dimensional, that is $\mathcal{P}(K)=0$.

Assume $\mathcal{P}(K) \neq 0$. Since $\mathcal{P}(K)$ is in ${ }_{\Gamma}^{\Gamma} \mathcal{Y} \mathcal{D}$, there are $g \in \Gamma, \chi \in \widehat{\Gamma}$ with $\mathcal{P}(K)_{g}^{\chi} \neq 0$. By [4, Lemma 3.1], we conclude $\chi(g) \neq 1$. But this is a contradiction, since for all $g \in \Gamma, \chi \in \widehat{\Gamma}$, $K{ }_{g}^{\chi} \neq 0$ implies $\chi(g)=1$. For, $K$ is the $\mathbb{k}$-span of all monomials

$$
\Psi\left(x_{\beta_{1}}^{N}\right) \cdots \Psi\left(x_{\beta_{m}}^{N}\right), \quad m \geqslant 1, \beta_{1}, \ldots, \beta_{m} \in \Phi_{I}^{+} .
$$

For any $\beta \in \Phi_{I}^{+}$there are natural numbers $b_{i}^{\beta}, 1 \leqslant i \leqslant \theta$, such that $\beta=\sum_{i=1}^{\theta} b_{i}^{\beta} \alpha_{i}$, where $\alpha_{1}, \ldots, \alpha_{\theta}$ are the simple roots. By (4.1), $x_{\beta} \in T\left(V_{I}\right)_{g_{\beta}}^{\chi_{\beta}}$, where

$$
g_{\beta}=\prod_{i \in I} g_{i}^{b_{i}^{\beta}}, \quad \chi_{\beta}=\prod_{i \in I} \chi_{i}^{b_{i}^{\beta}} .
$$

$4^{\mathrm{e}}$ SÉRIE - TOME $35-2002-\mathrm{N}^{\circ} 1$ 
Hence for all $\beta_{1}, \ldots, \beta_{m} \in \Phi_{I}^{+}$,

$$
\Psi\left(x_{\beta_{1}}^{N}\right) \cdots \Psi\left(x_{\beta_{m}}^{N}\right) \in K_{g}^{\chi}, \quad \text { where } \chi=\chi_{\beta_{1}}^{N} \cdots \chi_{\beta_{m}}^{N}, g=g_{\beta_{1}}^{N} \cdots g_{\beta_{m}}^{N} .
$$

It remains to show that $\chi(g)=1$.

Let $\alpha, \beta \in \Phi_{I}^{+}$. Since the braiding is of Cartan type,

$$
\chi_{\alpha}\left(g_{\beta}\right) \chi_{\beta}\left(g_{\alpha}\right)=\prod_{i, j} \chi_{i}^{b_{i}^{\alpha}}\left(g_{j}^{b_{j}^{\beta}}\right) \prod_{i, j} \chi_{j}^{b_{j}^{\beta}}\left(g_{i}^{b_{i}^{\alpha}}\right)=\prod_{i, j}\left(\chi_{i}\left(g_{j}\right) \chi_{j}\left(g_{i}\right)\right)^{b_{i}^{\alpha} b_{j}^{\beta}}=\prod_{i, j} \chi_{i}\left(g_{i}\right)^{a_{i j} b_{i}^{\alpha} b_{j}^{\beta}},
$$

and

$$
\chi_{\alpha}\left(g_{\alpha}\right)=\prod_{i} \chi_{i}\left(g_{i}\right)^{b_{i}^{\alpha} b_{i}^{\alpha}} \prod_{i<j}\left(\chi_{i}\left(g_{j}\right) \chi_{j}\left(g_{i}\right)\right)^{b_{i}^{\alpha} b_{j}^{\alpha}}=\prod_{i} \chi_{i}\left(g_{i}\right)^{b_{i}^{\alpha} b_{i}^{\alpha}} \prod_{i<j} \chi_{i}\left(g_{i}\right)^{a_{i j} b_{i}^{\alpha} b_{j}^{\alpha}} .
$$

Hence, since all the $\chi_{i}\left(g_{i}\right)$ have order $N$,

$$
\chi_{\alpha}^{N}\left(g_{\beta}^{N}\right) \chi_{\beta}^{N}\left(g_{\alpha}^{N}\right)=1, \quad \chi_{\alpha}^{N}\left(g_{\alpha}^{N}\right)=1
$$

Therefore we obtain

$$
\chi(g)=\prod_{i, j} \chi_{\beta_{i}}^{N}\left(g_{\beta_{j}}^{N}\right)=\prod_{i} \chi_{\beta_{i}}^{N}\left(g_{\beta_{i}}^{N}\right) \prod_{i<j} \chi_{\beta_{i}}^{N}\left(g_{\beta_{j}}^{N}\right) \chi_{\beta_{j}}^{N}\left(g_{\beta_{i}}^{N}\right)=1 .
$$

THEOREM 7.6. - Let A be a finite-dimensional pointed Hopf algebra with coradical $\mathbb{k} \Gamma$, and let $R$ be the diagram of $A$, that is

$$
\operatorname{gr} \mathcal{A} \simeq R \# \mathbb{k} \Gamma,
$$

and $R=\bigoplus_{n \geqslant 0} R(n)$ is a graded braided Hopf algebra in ${ }_{\Gamma}^{\Gamma} \mathcal{Y} \mathcal{D}$ with $R(0)=\mathbb{k} 1, R(1)=\mathcal{P}(R)$.

Assume that $R(1)$ is a Yetter-Drinfeld module of finite Cartan type with braiding $\left(b_{i j}\right)_{1 \leqslant i, j \leqslant \theta}$. For all $i$, let $q_{i}=b_{i i}, N_{i}=\operatorname{ord}\left(q_{i}\right)$. Assume that $\operatorname{ord}\left(b_{i j}\right)$ is odd and $N_{i}$ is not divisible by 3 and $>7$ for all $1 \leqslant i, j \leqslant \theta$.

(1) For any $1 \leqslant i \leqslant \theta$ contained in a connected component of type $B_{n}, C_{n}$ or $F_{4}$ resp. $G_{2}$, assume that $N_{i}$ is not divisible by 5 resp. by 5 or 7 .

(2) For any $1 \leqslant i, j \leqslant \theta$ and $i \nsim j$ assume $q_{i} q_{j}=1$ or $\operatorname{ord}\left(q_{i} q_{j}\right)=N_{i}$.

Then $R$ is generated as an algebra by $R(1)$, that is $A$ is generated by skew-primitive and group-like elements.

Proof. - Let $S:=R^{*}$ be the dual Hopf algebra of $R$ in the braided sense (see for example [3, Section 2]). $S=\bigoplus_{n \geqslant 0} S(n)$ is a graded braided Hopf algebra in $\Gamma_{\Gamma}^{\Gamma} \mathcal{Y D}$ with $S(0)=\mathbb{k} 1, S(n)=R(n)^{*}$, for all $n \geqslant 0$. By assumption there are $h_{i} \in \Gamma, \eta_{i} \in \widehat{\Gamma}, 1 \leqslant i, j \leqslant \theta$, with $b_{i j}=\eta_{j}\left(h_{i}\right)$ for all $i, j$, and a basis $\left(y_{i}\right)$ of $R(1)$ with $y_{i} \in R(1)_{h_{i}}^{\eta_{i}}$ for all $i$. Let $\left(x_{i}\right)$ in $V:=S(1)=R(1)^{*}$ be the dual basis of $\left(y_{i}\right)$. Then $x_{i} \in V_{g_{i}}^{\chi_{i}}$ with $\chi_{i}=\eta_{i}^{-1}, g_{i}=h_{i}^{-1}$ and $b_{i j}=\chi_{j}\left(g_{i}\right)=\eta_{j}\left(h_{i}\right)$ for all $1 \leqslant i, j \leqslant \theta$. Thus $V$ is a Yetter-Drinfeld module over $\Gamma$ with the same braiding as $R(1)$. By [5, Lemma 5.5], $R$ is generated by $R(1)$ if and only if $S(1)=\mathcal{P}(S)$. Hence by duality, $S$ is generated by $S(1)$, since $R(1)=\mathcal{P}(R)$. It is easy to see that $V=S(1) \subset \mathcal{P}(S)$. Hence there are canonical surjections of graded braided Hopf algebras

$$
T(V) \rightarrow S \rightarrow \mathfrak{B}(V)
$$


Here $T(V)$ is the tensor algebra, the elements $x_{i}$ are primitive and of degree one, and both maps are the identity on $V$. The kernel $I$ of the first map is a homogeneous ideal generated by elements of degree $\geqslant 2$, a coideal and stable under the action and coaction of $\Gamma$. Since $\mathfrak{B}(V)=T(V) / J$, where $J$ is the largest ideal with the same properties as $I$, there is a canonical surjection $S \rightarrow \mathfrak{B}(V)$.

The $x_{i}$ satisfy the Serre relations (4.6) by Lemma 7.2, and then the root vector relations (4.7) by Lemma 7.5. Therefore it follows from the description of $\mathfrak{B}(V)$ in Theorem 4.5 that $S=\mathfrak{B}(V)$. This means $S(1)=\mathcal{P}(S)$, hence by duality that $R$ is generated by $R(1)$.

A special case of the last theorem together with a main result in [5] allows to prove the following

COROLLARY 7.7. - Let $p>17$ be a prime number. Then any finite-dimensional pointed Hopf algebra with coradical $\mathbb{k}(\mathbb{Z} /(p))^{s}$ for some natural number $s$ is generated by group-like and skew-primitive elements.

Proof. - Let $A$ be a finite-dimensional pointed Hopf algebra with coradical $\mathbb{k}(\mathbb{Z} /(p))^{s}$ and let $R$ be the diagram of $A$. Then $R(1)$ is a Yetter-Drinfeld module of finite Cartan type by [5, Corollary 1.2]. Hence the claim follows from Theorem 7.6.

Let us state explicitly another corollary of the theorem.

COROLlary 7.8. - Under the hypothesis of Theorem 7.6, if the Dynkin diagram attached to the pointed Hopf algebra is connected, then $A$ is generated by group-like and skew-primitive elements.

In principle, the idea behind the proof of Theorem 7.6 is as follows. Let $A$ be a finitedimensional pointed Hopf algebra with coradical $\mathbb{k} \Gamma, \Gamma$ any finite group. Let $R$ be the diagram of $A$, and $S:=R^{*}$ the dual braided Hopf algebra. Consider the diagram $\widetilde{R}$ of the bosonization $S \# \mathbb{k} \Gamma$. Then $\mathcal{P}(S)$ is naturally embedded in $\mathcal{P}(\widetilde{R})$ (and this embedding is in fact an isomorphism). Moreover, $\operatorname{dim}(\mathcal{P}(R)) \leqslant \operatorname{dim}(\mathcal{P}(S)) \leqslant \operatorname{dim}(\mathcal{P}(\widetilde{R}))$, and $\operatorname{dim}(\mathcal{P}(R))=$ $\operatorname{dim}(\mathcal{P}(\widetilde{R}))$ if and only if $S(1)=\mathcal{P}(S)$ or $R=\mathfrak{B}(\mathcal{P}(R))$.

Corollary 7.7 can also be seen as a direct consequence of Section 6 and [5]: By [5] $\mathcal{P}(\widetilde{R})$ is of finite Cartan type. Then the result follows from Theorem 6.8 and 6.9 applied to $A=S \# \mathbb{k} \Gamma$.

The next theorem is another application of this principle. It shows that only very special dimensions are possible for finite-dimensional pointed Hopf algebras.

THEOREM 7.9. - For any finite group $\Gamma$ of odd order there is a natural number $n(\Gamma)$ such that the dimension of any finite-dimensional pointed Hopf algebra with coradical $\mathbb{k} \Gamma$ is $\leqslant n(\Gamma)$.

Proof. - Let $A$ be a finite-dimensional pointed Hopf algebra with coradical $\mathrm{k} \Gamma$ and diagram $R$ and $\widetilde{R}$ as defined above. Since $R$ and $\widetilde{R}$ are braided Hopf algebras over $\Gamma$ of the same dimension, and $\operatorname{dim}(\mathcal{P}(R)) \leqslant \operatorname{dim}(\mathcal{P}(\widetilde{R}))$, we can iterate this process and after finitely many steps we obtain a graded braided Hopf algebra $T$ over $\Gamma$ with $\operatorname{dim}(R)=\operatorname{dim}(T)$ and $T=\mathfrak{B}(\mathcal{P}(T))$. By a result of Graña [19] using [5, Theorem 3.1] which follows from [23], the number of isomorphism classes of Yetter-Drinfeld modules $V$ over the fixed group $\Gamma$ with finite-dimensional $\mathfrak{B}(V)$ is finite. Thus we can take for $n(\Gamma)$ the product of the largest such dimension with the order of $\Gamma$.

\section{Acknowledgements}

This work was partially supported by CONICET, Agencia Córdoba Ciencia-CONICOR, DAAD, the Graduiertenkolleg of the Math. Institut (Universität München) and Secyt (UNC). 


\section{REFERENCES}

[1] Andersen H.H., Jantzen J., Soergel W., Representations of quantum groups at a pth root of unity and of semisimple groups in characteristic p: Independence of p, Astérisque, Vol. 220, 1994.

[2] Andruskiewitsch N., DĂSCĂLEscu S, On quantum groups at -1, Algebr. Represent. Theory, to appear.

[3] Andruskiewitsch N., Graña M., Braided Hopf algebras over non-abelian groups, Bol. Acad. Ciencias (Córdoba) 63 (1999) 45-78, available at www.mate.uncor.edu/andrus/articulos.html.

[4] Andruskiewitsch N., Schneider H.-J., Lifting of quantum linear spaces and pointed Hopf algebras of order $p^{3}$, J. Algebra 209 (1998) 658-691.

[5] Andruskiewitsch N., Schneider H.-J., Finite quantum groups and Cartan matrices, Adv. in Math. 154 (2000) 1-45.

[6] Andruskiewitsch N., SCHneider H.-J., Lifting of Nichols algebras of type $A_{2}$ and pointed Hopf algebras of order $p^{4}$, in: Caenepeel S. (Ed.), Proceedings of the Colloquium in Brussels 1998, Hopf Algebras and Quantum Groups, Marcel Dekker, 2000, pp. 1-18.

[7] Andruskiewitsch N., Schneider H.-J., Pointed Hopf algebras, in: New Directions in Hopf Algebras, MSRI Series Cambridge Univ. Press, to appear.

[8] Beattie M., DĂSCĂLescu S., Grünenfelder L., On the number of types of finite-dimensional Hopf algebras, Inventiones Math. 136 (1999) 1-7.

[9] BeAtTie M., DĂSCĂLEscu S., RAiAnu S., Lifting of Nichols algebras of type B ${ }_{2}$, Preprint, 2001.

[10] CAenepeel S., DĂSCĂLescu S., Pointed Hopf algebras of dimension $p^{3}$, J. Algebra 209 (1998) $622-634$.

[11] Caenepeel S., DĂSCĂLescu S., Raianu S., Classifying pointed Hopf algebras of dimension 16, Comm. Algebra 28 (2000) 541-568.

[12] De Concini C., Procesi C., Quantum groups, in: D-modules, Representation Theory and Quantum Groups, Lecture Notes in Maths., Vol. 1565, Springer-Verlag, 1993, pp. 31-140.

[13] DiDT D., Linkable Dynkin diagrams, Preprint, 2001.

[14] Doi Y., Takeuchi M., Multiplication alteration by two-cocycles. The quantum version, Comm. Algebra 22 (14) (1994) 5715-5732.

[15] Drinfeld V.G., Quasi-Hopf algebras, Leningrad Math. J. 1 (1990) 1419-1457.

[16] GeLAKI S., On pointed Hopf algebras and Kaplansky's tenth conjecture, J. Algebra 209 (1998) 635657.

[17] Graña M., Pointed Hopf algebras of dimension 32, Comm. Algebra 28 (2000) 2935-2976.

[18] Graña M., On Pointed Hopf algebras of dimension $p^{5}$, Glasgow Math. J. 42 (2000) 405-419.

[19] Graña M., On Nichols algebras of low dimension, Contemp. Math. 267 (2000) 111-134.

[20] KaC V., Infinite Dimensional Lie Algebras, Third edition, Cambridge Univ. Press, 1995.

[21] Lusztig G., Finite dimensional Hopf algebras arising from quantized universal enveloping algebras, J. Amer. Math. Soc. 3, 257-296.

[22] Lusztig G., Quantum groups at roots of 1, Geom. Dedicata 35 (1990) 89-114.

[23] LuszTiG G., Introduction to Quantum Groups, Birkhäuser, 1993.

[24] Majid S., Crossed products by braided groups and bosonization, J. Algebra 163 (1994) 165-190.

[25] Masuoka A., Defending the negated Kaplansky conjecture, Proc. Amer. Math. Soc. 129 (2001) 3185-3192.

[26] Montgomery S., Hopf Algebras and Their Actions on Rings, CBMS Lecture Notes, Vol. 82, American Mathematical Society, 1993.

[27] Müller E., Some topics on Frobenius-Lusztig kernels, I, J. Algebra 206 (1998) 624-658.

[28] Musson I., Finite quantum groups and pointed Hopf algebras, Preprint, 1999.

[29] Nichols W.D., Bialgebras of type one, Comm. Algebra 6 (1978) 1521-1552.

[30] Nichols W.D., Zoeller M.B., A Hopf algebra freeness theorem, Amer. J. Math. 111 (1989) 381385.

[31] RADFORD D., Hopf algebras with projection, J. Algebra 92 (1985) 322-347.

[32] Ringel C., PBW-bases of quantum groups, J. Reine Angew. Math. 470 (1996) 51-88.

[33] Rosso M., Groupes quantiques et algèbres de battage quantiques, C.R.A.S. (Paris) 320 (1995) 145148. 
[34] Rosso M., Quantum groups and quantum shuffles, Inventiones Math. 133 (1998) 399-416.

[35] Schauenburg P., A characterization of the Borel-like subalgebras of quantum enveloping algebras, Comm. Algebra 24 (1996) 2811-2823.

[36] Stefan D., VAn Oystaeyen F., Hochschild cohomology and coradical filtration of pointed Hopf algebras, J. Algebra 210 (1998) 535-556.

[37] Woronowicz S.L., Differential calculus on compact matrix pseudogroups (quantum groups), Comm. Math. Phys. 122 (1989) 125-170.

(Manuscript received September 30, 1999; accepted, after revision, November 12, 2001.)

\section{Nicolás ANDRUSKIEWITSCH}

CIEM - CONICET

Facultad de Matemática, Astronomía y Física,

Universidad Nacional de Córdoba,

(5000) Ciudad Universitaria,

Córdoba, Argentina

E-mail: andrus@mate.uncor.edu

Hans-Jürgen SCHNEIDER

Mathematisches Institut,

Universität München,

Theresienstraße 39,

80333 München, Germany

E-mail: hanssch@rz.mathematik.uni-muenchen.de 